Copyright

by

Yi-Der Lin

2015 
The Dissertation Committee for Yi-Der Lin

certifies that this is the approved version of the following dissertation:

\section{The Vibrational Dynamics of 3D HOCl Above Dissociation}

Committee:

Linda Reichl, Supervisor

Arno Bohm

Greg O. Sitz

John F. Stanton

Philip J. Morrison 


\title{
The Vibrational Dynamics of 3D HOCl Above Dissociation
}

\author{
by \\ Yi-Der Lin, B.S; M.S
}

\author{
DISSERTATION \\ Presented to the Faculty of the Graduate School of \\ The University of Texas at Austin \\ in Partial Fulfillment \\ of the Requirements \\ for the Degree of \\ DOCTOR OF PHILOSOPHY
}

THE UNIVERSITY OF TEXAS AT AUSTIN

August 2015 
Dedicated to my parents. 


\section{Acknowledgments}

I would like to express the deepest appreciation to my committee chair Professor Linda Reichl, who has the attitude and the substance of a genius: her patience, flexibility, genuine caring and concern, and faith in me during the dissertation process enabled me to attend to life while also earning my Ph.D. Without her guidance and persistent help this dissertation would not have been possiblle. When I became too serious, her humor and friendly sarcasm allowed me to laugh and lightened my perspective. I am forever grateful.

I would like to give a heartfelt, special thanks to Prof. Christof Jung. He has been motivating, encouraging, and enlightening. I would like to thank Dr. Alexander Barr for guiding my research for the past few years and helping me to develop my background in dynamical system theory and numerical methods. Without his help and broad exploration in research this dissertation would not have been possible neither. I would also like to thank Dr. Kyungsun Na for supportive discussions. Special thanks to Dr. Yingyue Lee for helpful discussions. We thank R. Schinke for providing access to the PES used in this work. 


\title{
The Vibrational Dynamics of 3D HOCl Above Dissociation
}

\author{
Publication No. \\ Yi-Der Lin, Ph.D. \\ The University of Texas at Austin, 2015 \\ Supervisor: Linda Reichl
}

We have analyzed the vibrational dynamics of $\mathrm{HOCl}$ above dissociation using a 3D energy surface which governs the vibrational dynamics of $\mathrm{HOCl}$ above dissociation. The dynamics is dominated by an invariant manifold which is trasversally unstable for small spacing between $\mathrm{Cl}$ and $\mathrm{HO}$ complex, and stable for large spacing. Above dissocation, the InM separates two mirror image periodic orbits, embedded in a large chaotic sea, that can hold a large number of quantum states. The periodic orbits have the capability of forming significant quasibound states of the molecule above dissociation. 


\section{Table of Contents}

Acknowledgments $\quad$ v

Abstract vi

List of Figures $\quad$ ix

$\begin{array}{lll}\text { Chapter 1. Introduction } & 1\end{array}$

Chapter 2. The 3D Model of $\mathrm{HOCl} 5$

Chapter 3. Global Dynamics of 3D HOCl Above Dissociation 12

Chapter 4. Periodic Orbits of $\mathrm{HOCl} 21$

Chapter 5. The Invariant Manifold at $\theta=0, p_{\theta}=0 \quad 31$

5.1 Stability of the Invariant Manifold . . . . . . . . . . . . . . . . 35

5.1 .1 Unstable InM . . . . . . . . . . . . . . . . . 37

5.1 .2 Stable InM . . . . . . . . . . . . . . 41

Chapter 6. Conclusions 43

$\begin{array}{ll}\text { Appendices } & 45\end{array}$

Appendix A. Fractal scattering in a radiation field 46

A.1 Introduction . . . . . . . . . . . . . . . . . 46

A.2 The Hamiltonian . . . . . . . . . . . . . . . . . . . . . . . 47

A.3 Fractal Scattering . . . . . . . . . . . . . . . . . . 49

A.4 Symbolic Dynamics ................. 55

A.5 Conclusions ................... . . 61

Appendix B. Fractal Dimension of 2D and 3D HOCl $\quad 62$ 
Bibliography 


\section{List of Figures}

2.1 (a) Relationship between laboratory frame coordinates and Jacobi vectors $\mathbf{t}_{1}$ and $\mathbf{t}_{2}$ and $\theta$. (b) Body-fixed and laboratory axes differ by the angle $\beta$ so $\mathbf{t}_{1}$ always lies along the body $z$-axis.

2.2 The potential energy surface for $2 \mathrm{D} \mathrm{HOCl}$, as a function of $R$ and $\theta$, with $r$ fixed at $r=r_{e q}=1.85 \mathrm{~d}$.u.. The dashed line is the path of the $2 \mathrm{D} B_{o}$ periodic orbit for energy $E=$ 1.23 d.u. $=25,000 \mathrm{~cm}^{-1}$. The potential energy has values $V=0$ at $R=R_{e q}=3.23 \mathrm{~d} . u$. and $\theta=\theta_{e q}= \pm 1.34 \mathrm{rad}$ (the absolute minimum): $V=0.840$ d.u. $=17,053 \mathrm{~cm}^{-1}$ at $R=R_{s d}=$ 3.16 d.u. and $\theta=\theta_{s d}=0$ (the saddle point); $V=1.18$ d.u. $=$ $23,961 \mathrm{~cm}^{-1}$ at $R=4.03 \mathrm{~d} . u$. and $\theta=0$ (a local potential energy maximum). . . . . . . . . . . . . . .

3.1 The total energy is fixed at $E=1.231 \mathrm{~d} . \mathrm{u} .=25,000 \mathrm{~cm}^{-1}$. The energy of the incident chlorine atom is fixed at $E_{C l}=$ 1.034 d.u. $=21,000 \mathrm{~cm}^{-1}$. The remaining $E_{H O}=0.1969$ d.u. $=$ $4,000 \mathrm{~cm}^{-1}$ is distributed between rotation and vibration of $\mathrm{HO}$ so that $p_{\theta}=5.92 \mathrm{~d} . \mathrm{u}$. and vibration has amplitude in the range 1.622 d.u. $\leq r \leq 2.134$ d.u.. (a) Contour plot of scattered values of $p_{R}$ as a function of $\psi$ and $\chi$. (b) A plot of scattered values of $p_{R}$ as a function of $\chi$ for $\psi=\pi$. The greyscale in (a) ranges from 1 to 32, with 32 the lightest shade. Values of $p_{R}$ at the darkest and lightest points in (a) can be read from (b). $\left(p_{R}\right.$ in dimensionless units and $\chi$ in radians.) . . . . . . . .

3.2 The total energy is fixed at $E=1.231 \mathrm{~d} . \mathrm{u} .=25,000 \mathrm{~cm}^{-1}$. The energy of the incident chlorine atom is fixed at $E_{C l}=$ 1.034 d.u. $=21,000 \mathrm{~cm}^{-1}$. The remaining $E_{H O}=0.1969$ d.u. $=$ $4,000 \mathrm{~cm}^{-1}$ is distributed between rotation and vibration of $\mathrm{HO}$ so that $p_{\theta}=0.92$ d.u. and vibration has amplitude in the range 1.599 d.u. $\leq r \leq 2.159$ d.u.. (a) Contour plot of scattered values of $p_{R}$ as a function of $\psi$ and $\chi$. (b) A plot of scattered values of $p_{R}$ as a function of $\chi$ for $\psi=\pi$. The greyscale in (a) ranges from 1 to 32 , with 32 the lightest shade. Values of $p_{R}$ at the darkest and lightest points in (a) can be read from (b). $\left(p_{R}\right.$ in dimensionless units and $\chi$ in radians.) ......... 
3.3 (a) 2D scattering of HOCl. (b) 3D scattering of HOCl. Both the $\mathrm{HO}$ of $2 \mathrm{D}$ and $3 \mathrm{D}$ are released slightly excited at the asymptotic region. . . . . . . . . . . . . .

3.4 The scattering dynamics for the 3D molecule when total energy of the $\mathrm{Cl}+\mathrm{H}-\mathrm{O}$ system is $E=1.231 \mathrm{~d} . \mathrm{u} .=25,000 \mathrm{~cm}^{-1}$. The HO dimer has an initial total energy $E_{H O}=0.03261 .231 \mathrm{~d} . u .=$ $667 \mathrm{~cm}^{-1}$. The initial value of $p_{\theta}$ is $p_{\theta}=5.92 \mathrm{~d}$.u.. With this value of $p_{\theta}, \mathrm{HO}$ is essentially in its vibrational ground state initially. (a) A contour plot of scattered values of $p_{R}$ as a function of $\psi$ (vertical axis) and $\chi$ (horizontal axis). (b) A plot of scattered values of $p_{R}$ as a function of $\chi$ for $\psi=\pi$. Intervals of continuity and chaos are clearly seen. (c) A contour plot of scattered values of $p_{\theta}$ as a function of $\psi$ (vertical axis) and $\chi$ (horizontal axis). (d) A plot of scattered values of $p_{\theta}$ as a function of $\chi$ for $\psi=\pi$. The intervals of continuity and of discontinuity are the same as those of $p_{R}$, indicating that they are an intrinsic property of the molecule. All plots contain 1000 data points along the $\chi$ axis and 50 data points along the $\psi$ axis. The greyscale in (a) and (c) ranges from 1 to 32, with 32 the lightest shade. $\left(p_{R}\right.$ and $p_{\theta}$ in dimensionless units and $\chi$ and $\psi$ in radians.)

4.1 Surfaces of section for $2 \mathrm{D} \mathrm{HOCl}$. (a) $p_{R}$ versus $R$ when $\theta=$ $\theta_{\text {eq }}=1.34 \mathrm{rad}$ for $E=1.03$ d.u. $=21,000 \mathrm{~cm}^{-1}$. (b) $p_{R}$ versus $R$ when $\theta=\theta_{e q}$ for $E=1.23$ d.u. $=25,000 \mathrm{~cm}^{-1}$. (c) $p_{R}$ versus $R$ when $\theta=0$ for $E=1.03$ d.u. $=21,000 \mathrm{~cm}^{-1}$. (d) $p_{R}$ versus $R$ when $\theta=0$ for $E=1.23$ d.u. $=25,000 \mathrm{~cm}^{-1}$. (e) $p_{R}$ versus $R$ when $\theta=0$ for $E=1.03$ d.u. $=21,000 \mathrm{~cm}^{-1}$ in the neighborhood of the $B_{0}$ periodic orbit. (f) $p_{R}$ versus $R$ when $\theta=0$ for $E=1.23 \mathrm{~d} . u$. $=25,000 \mathrm{~cm}^{-1}$ in the neighborhood of the $B_{0}$ periodic orbit. . . . . . . . . . . . . . .

4.2 Bifurcation diagram of 3D $B_{0}$ stable (solid) and unstable (dashed). Both the period and energy are in the dimensionless units. . .

4.3 Sketch of path covered by periodic orbits in $x^{\prime}-z^{\prime}$ plane. (a) The $2 \mathrm{D}$ orbit $B_{0}$. (b) The stable $3 \mathrm{D} B_{0}$ orbit (solid) and the unstable $3 \mathrm{D}$ B0 orbit (dotted). (c) The $2 \mathrm{D}$ orbit $B_{1}$. (d) The $3 \mathrm{D}$ orbit $B_{1}$. Figures (c) and (d) look similar because the $\mathrm{HO}$ vibration is barely excited for the $3 \mathrm{D}$ orbit. . . . . . . . . .

4.4 Projected images of the $2 \mathrm{D} B_{0}$ (dashed), the stable $3 \mathrm{D} B_{0}$ (solid) periodic orbit, and the unstable 3D B0 orbit (dotted). (a) The $(R, \theta)$ plane. (b) The $(R, \theta, r)$ surface (3D orbits only). (c) The $R(t)$ versus $t$. (d) The $\left(p_{R}, R\right)$ plane. (e) The $\theta(t)$ versus $t$. (f) The $\left(p_{\theta}, \theta\right)$ plane. $(\mathrm{g})$ The $r(t)$ versus $t$ (3D $B_{0}$ only). (h) The $\left(p_{r}, r\right)$ plane $\left(3 \mathrm{D} B_{0}\right.$ only). . . . . . . . . . . 
4.5 Action of 3D $B_{0}$ (stable) orbit. The black solid line is the action derived from the relation of period and energy. Both the action and energy are in dimensionless units. . . . . . . . . . .

5.1 The potential energy and normal stability of the Invariant Manifold. The dark solid line in all figures is the MEP. (a) The potential energy $V_{I M}(R, r)$ for 2.75 d.u. $\leq R \leq 4.2$ d.u. and 1.45 d.u. $\leq r \leq 2.3 d$.u.. Contour lines are lines of constant potential energy. (b) The potential energy $V_{I M}(R, r)$ for 6.0 d.u. $\leq R \leq 8$ d.u. and 1.75 d.u. $\leq r \leq 1.90$ d.u. . Contour lines are lines of constant potential energy. (c) The variation of $\kappa$ as a function of $R$ and $r$ for 2.75 d.u. $\leq R \leq 4.2 d . u$. and 1.45 d.u. $\leq r \leq 2.3$ d.u.. In this region -1.85 d.u. $\leq \kappa \leq-$ $1.40 d . u$. and the InM is transversally unstable. The dashed line is the large period-five periodic orbit (see Fig. 5.2.a), with energy $E=1.23 \mathrm{~d} . u .=25,000 \mathrm{~cm}^{-1}$, on the InM. Contour lines are lines of constant $\kappa$. (d) The variation of $\kappa$ as a function of $R$ and $r$ for 6.0 d.u. $\leq R \leq 8.0$ d.u. and 1.75 d.u. $\leq r \leq 1.9$ d.u.. In this region $-0.03 d . u . \leq \kappa \leq 0.03 d . u$. and the InM is weakly stable. Contour lines are lines of constant $\kappa$. In all these plots, the dark solid line is the MEP. . . . . . . . . . . . . . .

5.2 Surfaces of Section of Birkhoff coordinates $\left(p_{R_{B}}, R_{B}\right)$ along the MEP on the Invariant Manifold each time a trajectory crossing the MEP in Fig. 5.1. These figures show numerous periodic orbits and a saddle point on the Invariant Manifold. In all three cases, the stable and unstable manifolds, associated with the saddle point, and the chaotic layer associated with them are plotted. (a) $E=1.23 \mathrm{~d} . u$. $=25,000 \mathrm{~cm}^{-1}$. The eleven black dots near the center show an effective SOS for the trajectory in Fig. 5.5 for time interval $200 \leq t \leq 600$. The inset shows the initial condition for echoes (see Fig. 5.3). (b) $E=1.48$ d.u. $=$ $30,000 \mathrm{~cm}^{-1}$. (c) $E=1.72$ d.u. $=35,000 \mathrm{~cm}^{-1}$. . . . . . . . .

5.3 (a) The density plot of 100,000 escaping trajectoris initially released in the inset of Fig. 5.2.a ( $\mathrm{t}$ in units of 1000 d.u.). (b) The flux, $P$ (in units of 1000 d.u.), of escaping trajectories (measured at $R=7.0$ d.u.) as a function of time $t$, shows echoes, as trajectories escape to the asymptotic region. The echoes have a period of about 180 d.u. . . . . . . . . . . . . . .

5.4 (a) The potential energy normal to the InM at $R=4.025$ d.u. and $r=r_{e q}=1.85 \mathrm{~d} . u$.. In this region the flow normal to the InM is hyperbolic, with stable and unstable manifolds normal to the InM. (b) Plot of $\theta$ versus $t$ for 100 initial conditions of the orbits with initial conditions taken a distance $\Delta \theta=10^{-7}$ from the large period-five orbit in Fig. 5.2.a. The slope of $\log (\theta)$ is $\omega=0.072$. 
5.5 Projections onto the coordinate axes for two orbits with energy $E=1.23$ d.u. $=25,000 \mathrm{~cm}^{-1}$ close to the stable manifold of the unstable InM in the neighborhood of the ring of numbered black dots in Fig. 5.2.a. The trajectories have different long time behavior. (a) $R(t)$ versus $t$. (b) $\theta(t)$ versus $t$. (c) $r(t)$ versus $t . \ldots \ldots \ldots \ldots \ldots$

A.1 A Poincaré surface of section with $p$ and $x$ plotted each time $\phi=\pi / 2$. (a) The curves $\mathrm{n}-\mathrm{u}$ and $\mathrm{s}-\mathrm{n}$ ( $\mathrm{r}-\mathrm{p}$ and $\mathrm{p}-\mathrm{v}$ ) are segments of the unstable and stable manifolds of $F_{L}\left(F_{R}\right)$, respectively. The fundamental region is formed by the curves n-q, q-p, n-m and m-p. (b) The segments of the stable manifolds r-p (bluesolid line) and n-s (red dashed line) are integrated backward in time for four iterations of the PSS.

A.2 (a) The points labeled $\alpha_{-5}, \ldots ., \alpha_{+3}$ show the movement of a single point, $\alpha_{n}$, incident from the right for nine iterations of the PSS. Initially, the point lies in a tangle in the asymptotic region indicated by the shaded region. The point $\alpha$ takes two steps inside the fundamental area $R$ as the PSS is iterated forward in time, and eventually the entire shaded region is mapped back to the right and all points in it are reflected. (b) The points labeled $\beta_{-5}, \ldots, \beta_{+4}$ show the movement of a single point, $\beta_{n}$, incident from the left for ten iterations of the PSS. The point $\beta$ takes two steps inside the fundamental region before being reflected back to the left. . . . . . . . . . . . . . . .

A.3 (a) The evolution in time of a line of initial conditions for a particle incident from the left. The plot shows four iterations of the PSS. One more iteration would show the line of points stepping inside the fundamental region $R$. (b) The delay time (the time each point is delayed before being transmitted (red) or reflected (blue)) for the line of initial points shown in (a). (c) A plot of number of steps taken inside the fundamental region before being transmitted (red) or reflected (blue), for those trajectories that take fewer than twenty steps inside $R$. A complete plot would show step numbers ranging from one to infinity and a fractal mixed structure of transmitted and reflected points. ................. 
A.4 (a) The first iteration of the stable manifolds backward in time. The shaded regions are basins of the asymptotic regions that cut through the fundamental region $R$ on the first iteration of the PSS. The shaded regions cut two segments out of the unstable manifold of $F_{L}$ and cut one segment out of the unstable manifold of $F_{R}$. The three remaining pieces of the unstable manifold of $F_{L}$ are labeled "A", "B", and "C". The two remaining pieces of the unstable manifold of $F_{R}$ are labeled "+" and "C". (b) The second iteration of the stable manifolds backward in time. The left-most symbols of the unstable manifold of $F_{L}$ are "A", "B", and "C". The remaining segments are labeled "B", "_", "+", and "C". The two segments of the unstable manifold of $F_{L}$ are now cut into four segments that are labeled "A", "A",

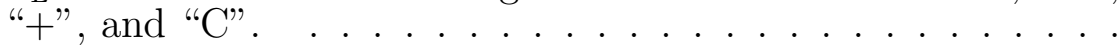

A.5 (a) The branching tree for symbolic dynamics associated with scattering from the left. (b) The branching tree for scattering from the right. . . . . . . . . . . . . .

B.1 Scattering dynamics of the 3D Cl-HO system. (a) Magnification of Fig. 3.4.b (5000 data points for $\chi$ ). (b) Magnification of the left discontinuous region of Fig. B.1.a. (c) Magnification of the right discontinuous region of Fig. B.1.a. $\left(p_{R}\right.$ in dimensionless units and $\chi$ in radians.) . . . . . . . . . . . .

B.2 Scattering dynamics of the 2D Cl-HO system. (a) A plot of $p_{R}$ versus $\chi$ for the case is which the bond length of the $\mathrm{HO}$ dimer is held fixed at the equilibrium value $r_{e q}=1.8389$ d.u., $p_{\theta}=5.92$ d.u., and the total energy is fixed at $E=1.231$ d.u. $=$ $25,000 \mathrm{~cm}^{-1}$ (5000 data points for $\chi$ ). (b) Magnification of left discontinuous region in Fig. 4.a. (c) Magnification of the right discontinuous region in Fig. 4.a. ( $p_{R}$ in dimensionless units and $\chi$ in radians.) . . . . . . . . . . . . . . .

B.3 Running time plots for values of $\chi$ in the neighborhood of the mirror point B. Note that these plots contain the time for $\mathrm{Cl}$ to travel from $R=12$ d.u. to the reaction region, then interact with $\mathrm{HO}$ and return to $R=12$ d.u.. (a) $3 \mathrm{D}$ molecule. (b) $2 \mathrm{D}$ molecule. ( $\tau$ in dimensionless units and $\chi$ in radians. $). . .$. 


\section{Chapter 1}

\section{Introduction}

One of the most active regions of a molecule involves the vibrational dynamics of its atomic constituents just above the dissociation energy of the molecule. The vibrational dynamics determines the pathways for dissociation and recombination of the constituents of the molecule.

However, little is known about these dynamical processes because they have a large number of degrees of freedom, and the nonlinear classical and quantum dynamics of conservative systems with more than two degrees of freedom ${ }^{1}$ is not well understood. Recent studies of the classical nonlinear vibrational dynamics of small molecules, above the dissociation energy, has revealed bifurcations and dynamical structures that can have significant influence on the dissociation dynamics of the molecule [1-4]. Therefore, small molecules provide a perfect laboratory for studying the classical-quantum correspondence in systems with more than two degrees of freedom.

Recent work on the classical-quantum correspondence of dynamical processes in models of $\mathrm{HOCl}$ with two degrees of freedom $(2 \mathrm{D})[5,6]$ has shown

\footnotetext{
${ }^{1}$ This dissertation is based on the author's three papers $[15,18,19]$ with coauthors Linda Reichl, Christof Jung, Alexandar Barr, and Kyungsun Na. They all contribute to published papers importantly and equally.
} 
that quasibound states (long-lived quantum states above dissociation), find their support on classical periodic orbits that can exist above the $\mathrm{HO}-\mathrm{Cl}$ dissociation energy. Periodic orbits provide a pathway along which a quantum wave can constructively interfere with itself and thereby create a bound or quasibound quantum state. The connection between classical periodic orbits and quantum bound and quasibound states is well established for 2D systems $[5,7]$, and there is evidence [4] that a similar correspondence exists for systems with three degrees of freedom (3D).

When dealing with scattering problems with three degrees of freedom (3D), surfaces of section (SOS) become four dimensional and one cannot visualize the complexity of the scattering processes in the same way [8], although the scattering process is still dominated by the normal hyperbolic invariant manifold (NHIM) of the outer fixed point of the SOS. Studies of fractal scattering processes have focused on systems with two degrees of freedom (2D) because in 2D they are easy to visualize [9-15] and characterize using Poincaré surfaces of section (SOS). In 2D systems it is possible to follow the flow of stable and unstable manifolds, as they form an increasingly complex network of tendrils in the phase space. As we show in Appendix A, these tendrils can be categorized in terms of symbolic dynamics and their fractal structure then becomes apparent.

In subsequent sections, we analyze the scattering dynamics of $\mathrm{Cl}$ and $\mathrm{HO}$ just above the dissociation energy of the $\mathrm{HOCl}$ molecule. Our analysis of Cl-HO scattering dynamics uses a potential energy surface (PES), constructed 
by Weiss et al. [16], that realistically governs the dynamics of the bound $\mathrm{HOCl}$ molecule, and the $\mathrm{Cl}-\mathrm{HO}$ scattering system for an interval of energies just above dissociation of $\mathrm{Cl}$ from $\mathrm{HO}$. For the 3D Cl-HO scattering process, we find behavior similar to that observed by Jung et al. [17], with the vibrational degree of freedom of $\mathrm{HO}$ playing the role of a weakly coupled third degree of freedom (at least for some parameter regimes). For a given initial energy of $\mathrm{Cl}$ and $\mathrm{HO}$, the initial conditions of both objects can be specified in terms of two initial "phases" (which we define later). This provides a "landscape" that shows a range of initial phases for which the scattering function (outgoing momentum versus initial phases) appears to have fractal structure.

This fractal structure is formed by the intersection of the stable manifold of the outer NHIM ( which extends into the asymptotic region ) with the torus formed by the initial phases of $\mathrm{Cl}$ and $\mathrm{HO}$ in the asymptotic region. When a $\mathrm{Cl}$ trajectory has an initial phase that lies on the manifolds of NHIM in the asymptotic region, the subsequent trajectory of $\mathrm{Cl}$ will have an infinite delay time and its outgoing momentum will approach zero in the asymptotic region as the trajectory approaches the outer fixed point (which by definition has zero momentum). These singular points form a fractal structure which is an intrinsic property of the molecule, and all other dynamical quantities will exhibit this same fractal behavior.

Additionally, we explore the phase space dynamics of a 3D model of $\mathrm{HOCl}$ above dissociation to locate specific structures that can support significant quasibound states in the quantum system. We will show the fractal 
structure of the scattering dynamics of $\mathrm{Cl}$ and $\mathrm{HO}[18]$ and then focus on the phase space dynamics in the interior of $\mathrm{HOCl}$ above dissociation [19].

We begin in Sect. 2, with a description of the coordinates and Hamiltonian used to describe the dynamics of 3D HOCl. In Sect. 3, we compare the global vibrational dynamics of $2 \mathrm{D}$ and $3 \mathrm{D}$ models of $\mathrm{HOCl}$ and in Sect. 4 locate the dominant periodic orbits above dissociation. In Sect. 5, we discuss the properties of the Invariant Manifold that dominates the $\mathrm{HOCl}$ vibrational dynamics. Finally, in Sect. 6, we make some concluding remarks.

In Appendix A, we demonstrate the concepts used to analyze the dynamics of $3 \mathrm{D} \mathrm{HOCl}$, but on a simpler system in a driven inverted Gaussian. The dynamical system in Appendix A has one and half degrees of freedom. There are two unstable fixed points in (the positive and negative asymptotic regions) on the Poincaré Surfaces of Section (SOS). Appendix A demonstrates the application of Liouville's theorem, the idea that area conservation can be identified on a SOS. The tendrils from the stable and unstable manifolds and their intersections provide a good tool to identify the boundaries of conserved areas and visualize the flow of area. We focus on the heteroclinic intersections between these two unstable fixed points and map out the regular pattern of flow on the SOS by the concept of Conservation of Area. We further classify the fractal pattern of the flow which brings the phase space area into either positive or negative infinities as a function of mapping time. This pattern leads us to build up a dynamical tree to describe the mapping and be able to calculate the topological entropy exactly. 


\section{Chapter 2}

\section{The 3D Model of $\mathrm{HOCl}$}

The $\mathrm{HOCl}$ molecule consists of strongly bound hydrogen and oxygen atoms $(\mathrm{HO})$ and a chlorine atom $(\mathrm{Cl})$ more weakly bound to the $\mathrm{HO}$ system. In its bound configuration, the $\mathrm{HOCl}$ molecule consists of $\mathrm{H}, \mathrm{O}$, and $\mathrm{Cl}$ atoms, with masses $m_{H}, m_{O}$, and $m_{C l}$, respectively. The molecule dissociates into a free $\mathrm{Cl}$ atom and a bound $\mathrm{HO}$ molecule above $E=20,312 \mathrm{~cm}^{-1}[16,20]$. The classical dynamics of the $\mathrm{HOCl}$ system has been discussed in [14] for a

$2 \mathrm{D}$ version of the molecule in which the $\mathrm{HO}$ bond is held fixed. As in [14], we introduce lab coordinates $\left(x^{\prime}, y^{\prime}, z^{\prime}\right)$ and body-fixed coordinates $(x, y, z)$ whose origin is the center of mass of the molecule. A sketch of $\mathrm{HOCl}$ relative to the lab and body frames is shown in Fig. 2.1. The total angular momentum of the molecule, $\mathbf{L}_{t o t}$, is conserved. We assume that all of the dynamics occurs in the $\left(x^{\prime}, z^{\prime}\right)$ and $(x, z)$ planes and that any angular momentum vectors generated by internal rotations of the molecule lie along parallel $y^{\prime}$ and $y$ axes in the lab and body-fixed frames, respectively.

A vector $\mathbf{t}_{1}$ of length $R$, connects the center of mass of $\mathrm{HO}$ to $\mathrm{Cl}$, and a vector $\mathbf{t}_{2}$ of length $r$ connects $\mathrm{H}$ to $\mathrm{O}$ ( $R$ and $r$ are Jacobi coordinates). The angle between $\mathbf{t}_{1}$ and $\mathbf{t}_{2}$ is $\theta$ where $\theta=0$ for the linear configuration $\mathrm{H}-\mathrm{O}-\mathrm{Cl}$. 


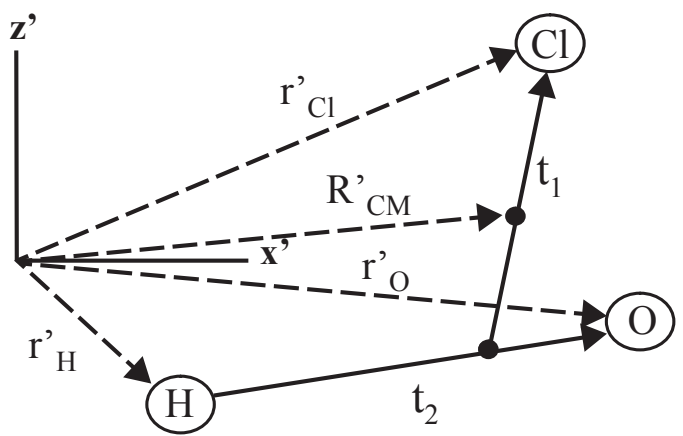

(a)

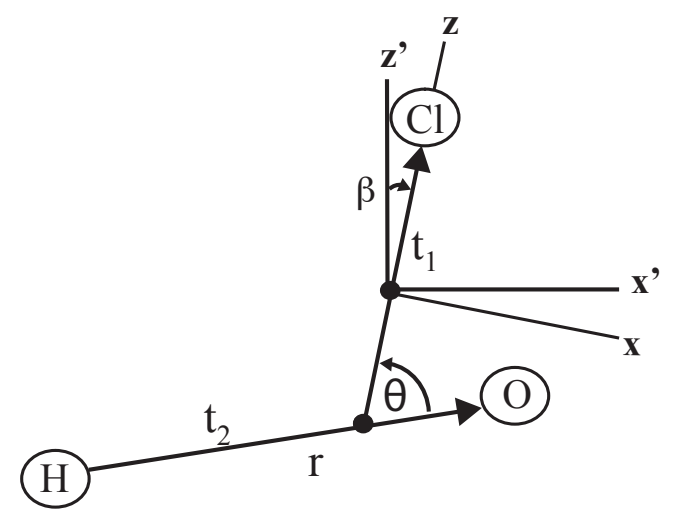

(b)

Figure 2.1: (a) Relationship between laboratory frame coordinates and Jacobi vectors $\mathbf{t}_{1}$ and $\mathbf{t}_{2}$ and $\theta$. (b) Body-fixed and laboratory axes differ by the angle $\beta$ so $\mathbf{t}_{1}$ always lies along the body $z$-axis. 
The center of mass of the molecule lies along $\mathbf{t}_{1}$ a distance $\frac{m_{d}}{M} R$ from the $\mathrm{Cl}$ atom, where $m_{d}=m_{O}+m_{H}, M=m_{C l}+m_{O}+m_{H}$. Let $\mathbf{r}_{C l}^{\prime}, \mathbf{r}_{H}^{\prime}$, and $\mathbf{r}_{O}^{\prime}$ denote the displacement of the $\mathrm{Cl}, \mathrm{H}$, and $\mathrm{O}$ atoms from the lab frame origin. Let $\mathbf{R}_{c m}=\left(m_{C l} \mathbf{r}_{C l}^{\prime}+m_{O} \mathbf{r}_{O}^{\prime}+m_{H} \mathbf{r}_{H}^{\prime}\right) / M$ denote the displacement of the center of mass of $\mathrm{HOCl}$ from the lab frame origin. The kinetic energy of $\mathrm{HOCl}$ is given by

$$
T=\frac{M}{2} \dot{\mathbf{R}}_{c m}^{2}+\frac{\mu_{1}}{2} \dot{\mathbf{t}}_{1}^{2}+\frac{\mu_{2}}{2} \dot{\mathbf{t}}_{2}^{2}
$$

where $\mu_{1}=\frac{m_{C l} m_{d}}{M}=\frac{595}{52} u$ and $\mu_{2}=\frac{m_{H} m_{O}}{m_{d}}=\frac{16}{17} u$ ( $u$ the atomic mass unit). The total angular momentum is given by $\mathbf{L}_{t o t}=\mathbf{L}_{1}+\mathbf{L}_{2}$ where $\mathbf{L}_{1}=\mu_{1} \mathbf{t}_{1} \times \dot{\mathbf{t}}_{1}$ and $\mathbf{L}_{2}=\mu_{2} \mathbf{t}_{2} \times \dot{\mathbf{t}}_{2}$.

We assume that $\mathbf{t}_{1}$ lies along the body $z$-axis and that the body $y$-axis is directed perpendicular to the plane of the molecule. We can then write $\mathbf{t}_{1}=R \hat{\mathbf{z}}$ and $\mathbf{t}_{2}=r \sin (\theta) \hat{\mathbf{x}}+r \cos (\theta) \hat{\mathbf{z}}$. We further assume that the body frame $(x, z)$ axes make an angle $\beta$ with respect to the lab frame $\left(x^{\prime}, z^{\prime}\right)$ axes so that if the two frames rotate relative to one another, the angular velocity of rotation is $\dot{\beta} \hat{\mathbf{y}}$.

The Hamiltonian for the $\mathrm{HO}+\mathrm{Cl}$ system (dropping the center of mass motion) can then be written in the form

$$
H=\frac{p_{R}^{2}}{2 \mu_{1}}+\frac{p_{r}^{2}}{2 \mu_{2}}+\frac{p_{\theta}^{2}}{2 \mu_{2} r^{2}}+\frac{p_{\theta}^{2}}{2 \mu_{1} R^{2}}+\frac{p_{\beta}^{2}}{2 \mu_{1} R^{2}}-\frac{p_{\theta} p_{\beta}}{\mu R^{2}}+D_{e} V(R, \theta, r)=E,
$$

where $E$ is the total energy. The quantity $D_{e} V(R, \theta, r)$ is the potential energy and $D_{e}=20,312.3 \mathrm{~cm}^{-1}=2.518 \mathrm{eV}$ is the energy at which $\mathrm{Cl}$ dissociates from HO. The canonical momenta $p_{R}, p_{r}, p_{\theta}$, and $p_{\beta}$ can be written $p_{R}=\mu_{1} \dot{R}$, 
$p_{r}=\mu_{2} \dot{r}, p_{\theta}=\mu_{2} r^{2}(\dot{\theta}+\dot{\beta})$ and $p_{\beta}=\mu_{2} r^{2}(\dot{\theta}+\dot{\beta})+\mu_{1} R^{2} \dot{\beta}$. The total angular momentum of the molecule is then given by

$$
\mathbf{L}_{t o t}=\mathbf{L}_{1}+\mathbf{L}_{2}=p_{\beta} \hat{\mathbf{y}}
$$

where

$$
\mathbf{L}_{1}=\mu_{1} \mathbf{t}_{1} \times \dot{\mathbf{t}}_{1}=\mu_{1} R^{2} \dot{\beta} \hat{\mathbf{y}} \quad \text { and } \quad \mathbf{L}_{2}=\mu_{2} \mathbf{t}_{2} \times \dot{\mathbf{t}}_{2}=\mu_{2} r^{2}(\dot{\theta}+\dot{\beta}) \hat{\mathbf{y}}
$$

We will consider that the dynamics of the molecule for the case $\mathbf{L}_{t o t}=p_{\beta} \hat{\mathbf{y}}=0$ So

$$
\dot{\beta}=-\frac{\mu_{2} r^{2} \dot{\theta}}{\left(\mu_{2} r^{2}+\mu_{1} R^{2}\right)} .
$$

Note that $\mathbf{L}_{1}$ and $\mathbf{L}_{2}$ need not be zero so that $\mathrm{Cl}$ and $\mathrm{HO}$ can rotate relative to one another.

Let us now write the above quantities in terms of dimensionless units (d.u.). We parametrize all energies in units of $D_{e}$, lengths in units of the Bohr radius $a_{B}=5.2917 \times 10^{-11} \mathrm{~m}$, and angular momenta in terms of Planck's constant $\hbar=1.05457 \times 10^{-34} \mathrm{~J} \cdot \mathrm{s}$. Then $H=D_{e} H^{\prime}, E=D_{e} E^{\prime}, R=a_{B} R^{\prime}$, $r=a_{B} r^{\prime}, p_{R}=\frac{\hbar}{a_{B}} p_{R}^{\prime}, p_{r}=\frac{\hbar}{a_{B}} p_{r}^{\prime}, p_{\theta}=\hbar p_{\theta}^{\prime}, p_{\beta}=\hbar p_{\beta}^{\prime}$, and time $t=\frac{\hbar}{D_{e}} t^{\prime}$, where primed quantities are dimensionless. If we now drop the primes on dimensionless quantities, the (dimensionless) Hamiltonian takes the form

$$
H=\frac{p_{R}^{2}}{2 \delta_{1}}+\frac{p_{r}^{2}}{2 \delta_{2}}+\frac{p_{\theta}^{2}}{2 \delta_{2} r^{2}}+\frac{p_{\theta}^{2}}{2 \delta_{1} R^{2}}+V(R, \theta, r)=E,
$$

where $\delta_{1}=\frac{\mu_{1} D_{e} a_{B}^{2}}{\hbar^{2}}=1930.3$ d.u. and $\delta_{2}=\frac{\mu_{2} D_{e} a_{B}^{2}}{\hbar^{2}}=158.79$ d.u.. The quantity $V(R, \theta, r)$ is the potential energy in dimensionless units (d.u.). Equation (2.6) describes the dynamics of a system with three degrees of freedom (3D). 
The potential energy surface (PES), $V(R, \theta, r)$, used in this analysis was constructed by Weiss et al. [16] and governs the dynamics of the $\mathrm{HOCl}$ molecule and the $\mathrm{Cl}$ and $\mathrm{HO}$ system for a region of energies below and just above dissociation of $\mathrm{Cl}$ from HO. This PES can be written in the form

$$
V\left(R_{O C l}, R_{H O}, R_{C l H}\right)=V_{I}\left(R_{O C l}, R_{H O}, R_{C l H}\right)+V_{H O}\left(R_{H O}\right) .
$$

where $R_{O C l}, R_{H O}$ and $R_{C l H}$ are the distances between $\mathrm{O}$ and $\mathrm{Cl}, \mathrm{H}$ and $\mathrm{O}$, and $\mathrm{Cl}$ and $\mathrm{H}$, respectively (in dimensionless units), and

$$
V_{H O}\left(R_{H O}\right)=1.835\left[1-\mathrm{e}^{-\beta_{H O}\left(R_{H O}-\bar{R}_{H O}\right)}\right]^{2},
$$

is the potential energy of the $\mathrm{HO}$ system in the asymptotic region with $\beta_{H O}=$ 1.2139 d.u. and $\bar{R}_{H O}=1.8323$ d.u.. The potential energy $V_{I}\left(R_{O C l}, R_{H O}, R_{C l H}\right)$ is the interaction energy of $\mathrm{Cl}$ and $\mathrm{HO}$ and has the form

$$
\begin{array}{r}
V_{I}\left(R_{O C l}, R_{H O}, R_{C l H}\right)=\frac{1}{2}\left[1+\tanh \left(6-R_{C l O}\right)\right] \\
\sum_{i=0}^{7} \sum_{j=0}^{7} \sum_{\ell=0}^{7} a_{i, j, \ell} g_{i}\left(R_{H O}\right) h_{j}\left(R_{O C l}\right) d_{\ell}\left(R_{C l H}\right),
\end{array}
$$

where

$$
\begin{gathered}
g_{i}\left(R_{H O}\right)=\left[1-\mathrm{e}^{-k_{H O}\left(R_{H O}-\bar{R}_{H O}\right)}\right]^{i}, \\
h_{j}\left(R_{O C l}\right)=\left[1-\mathrm{e}^{-k_{O C l}\left(R_{O C l}-\bar{R}_{O C l}\right)}\right]^{j+1}, \\
d_{\ell}\left(R_{C l H}\right)=\left[1-\mathrm{e}^{-k_{C l H}\left(R_{C l H}-\bar{R}_{C l H}\right)}\right]^{\ell}, \\
\bar{R}_{H O}=1.8323 \text { d.u., } \bar{R}_{O C l}=3.2 \text { d.u., } \bar{R}_{C l H}=4 \text { d.u., } k_{H O}=0.3 \text { d.u., } k_{O C l}=0.8 \\
\text { d.u., and } k_{C l H}=0.1 \text { d.u.. Note that } R_{H O}=r \text { and the distances } R_{O C l}, R_{H O}
\end{gathered}
$$


and $R_{C l H}$ are related to $R, r$, and $\theta$ by the triangle equations:

$$
R_{O C l}=\sqrt{\left(\frac{m_{H}}{m_{d}}\right)^{2} r^{2}+R^{2}-2\left(\frac{m_{H}}{m_{d}}\right) r R \cos (\theta)}
$$

and

$$
R_{C l H}=\sqrt{\left(\frac{m_{O}}{m_{d}}\right)^{2} r^{2}+R^{2}+2\left(\frac{m_{O}}{m_{d}}\right) r R \cos (\theta)} .
$$

The ground state (lowest bound state) energy for this system is $E=0$ d.u. The dissociation energy for $\mathrm{Cl}$ from $\mathrm{HO}$, when rotation and vibration energy of $\mathrm{HO}$ is zero is $E=D_{e}=1.0 \mathrm{~d} . \mathrm{u}$. The $2 \mathrm{D}$ model of $\mathrm{HOCl}$ is obtained by setting $r=\bar{R}_{H O}$ and $p_{r}=0$ in Eq. (2.6) and $R_{H O}=\bar{R}_{H O}$ in Eqs. (2.8) and (2.10). This PES has been used by several authors $[14,16,18]$ to describe the dynamical behavior of $\mathrm{HOCl}$. 


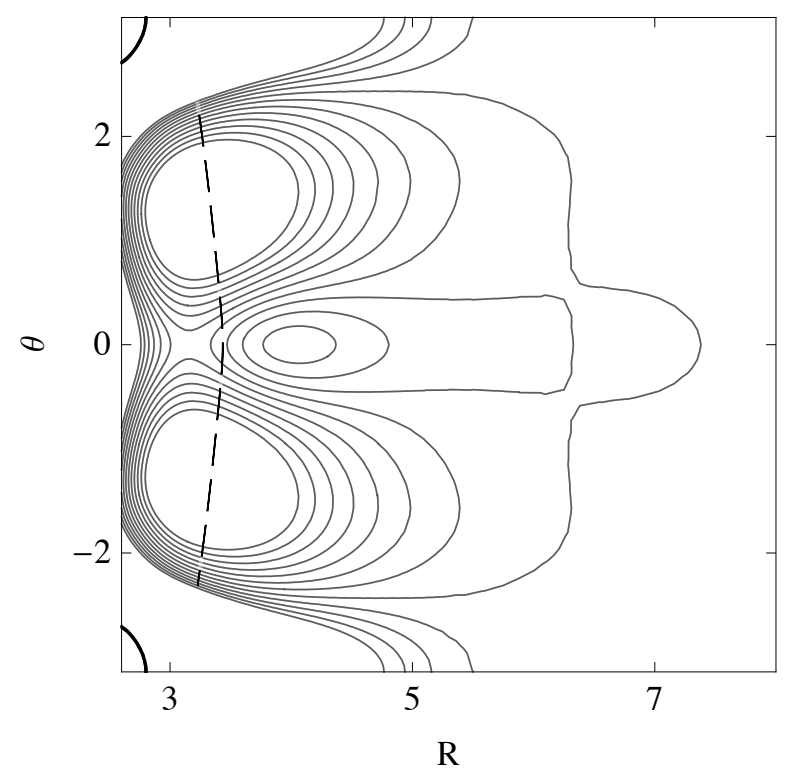

Figure 2.2: The potential energy surface for 2D $\mathrm{HOCl}$, as a function of $R$ and $\theta$, with $r$ fixed at $r=r_{e q}=1.85$ d.u. . The dashed line is the path of the $2 \mathrm{D} B_{o}$ periodic orbit for energy $E=1.23 \mathrm{~d} . u$. $=25,000 \mathrm{~cm}^{-1}$. The potential energy has values $V=0$ at $R=R_{e q}=3.23 \mathrm{~d} . u$. and $\theta=\theta_{e q}= \pm 1.34 \mathrm{rad}$ (the absolute minimum): $V=0.840 \mathrm{~d} . u$. $=17,053 \mathrm{~cm}^{-1}$ at $R=R_{s d}=3.16 \mathrm{~d}$.u. and $\theta=\theta_{s d}=0$ (the saddle point); $V=1.18 d . u .=23,961 \mathrm{~cm}^{-1}$ at $R=4.03 \mathrm{~d}$.u. and $\theta=0$ (a local potential energy maximum). 


\section{Chapter 3}

\section{Global Dynamics of 3D HOCl Above Dissociation}

In the 3D model of $\mathrm{HOCl}$, the energy and the total angular momentum of the $\mathrm{HOCl}$ system are constants of the motion, and both the HO rotation and vibration participate in the dynamics of the scattering process. For energies just above dissociation, $\mathrm{Cl}$ impinges on the $\mathrm{HO}$ complex and scatters from it, leaving both the $\mathrm{Cl}$ atom and the $\mathrm{HO}$ complex in an altered state. In order to study this scattering process, we must specify initial conditions for both the $\mathrm{Cl}$ atom and the $\mathrm{HO}$ complex.

We can distribute the initial total energy $E$ (which is conserved) be-

tween the incident energy of $\mathrm{Cl}, E_{C l}=\frac{p_{R}^{2}}{2 \delta_{1}}$, and the rotational and vibration energies of HO, $E_{\text {rot }}$ and $E_{v i b}$, so that $E=E_{C l}+E_{r o t}+E_{v i b}$. The initial value of $E_{C l}$ fixes the initial value of $p_{R}$. The remaining energy is distributed between $E_{\text {rot }}$ and $E_{v i b}$ asymptotically. The angular momentum of HO relative to its center of mass is given by $\mathbf{L}_{2}=p_{\theta} \hat{\mathbf{y}}$. For a given total energy $E$, in the asymptotic region $\left(R_{\text {in }}=R_{\text {out }}=12\right.$ d.u. $)$, we can specify a range of initial values of $E_{C l}$. We also specify a range of initial values for the angular position $\chi=\theta$ and vibrational phase $\psi$ in the asymptotic region. 
The phase $\chi=\theta$ is the initial angular position of the HO dimer at $R_{\text {in }}=$ 12 d.u. The phase $\psi$ is determined by the initial vibrational configuration of $\mathrm{HO}$ in the asymptotic region and ranges over all possible initial configurations of $\mathrm{HO}$ at its given initial energy. It is determined as follows. In the asymptotic regime the Hamiltonian of $\mathrm{HO}$ is given by

$$
H_{H O}=\frac{p_{r}^{2}}{2 \delta_{2}}+\frac{p_{\theta}^{2}}{2 \delta_{2} r^{2}}+V_{H O}(r)=E_{r o t}+E_{v i b}=E_{H O}
$$

The value of $p_{\theta}$ and the total energy of $\mathrm{HO}$ are fixed, and the initial values of $\left(p_{r}, r\right)$ are allowed to range over one complete oscillation of the $\mathrm{HO}$ vibration at that total energy. The phase $\psi$ ranges over the interval $0 \leq \psi \leq 2 \pi$ as $\mathrm{HO}$ goes through one complete oscillation. Each initial condition can therefore be labeled uniquely by $\left(E, p_{R}, p_{\theta}, \chi, \psi\right)$.

For a given value of total energy $E$, we can plot values of $p_{R}$ and $p_{\theta}$ after the scattering process has occurred for ranges of initial phases $0 \leq \chi \leq 2 \pi$ and $0 \leq \psi \leq 2 \pi$ and different incident $\mathrm{Cl}$ energies $E_{C l}$. We will analyze scattering properties for a subspace of the scattering process with total angular momentum zero and total energies in the interval 1.034 d.u. $\leq E \leq 1.231$ d.u. $\left(21,000 \mathrm{~cm}^{-1} \leq E \leq 25,000 \mathrm{~cm}^{-1}\right)$. The PES that we use was developed to represent the dynamics of $\mathrm{HOCl}$ above dissociation for these, and slightly higher energies. The subspace with total angular momentum zero gives us a good picture of the nature of the scattering processes. We do not expect it to change significantly for non-zero total angular momentum.

In the scattering process, the total energy is distributed between the 
rotational and vibrational energy of $\mathrm{HO}$ and the incident energy of $\mathrm{Cl}$ in different ways. If $\mathrm{Cl}$ approaches $\mathrm{HO}$ with its momentum directed along a line through the center of mass of $\mathrm{HO}$, then its impact parameter is zero and $\mathrm{HO}$ does not rotate. If $\mathrm{Cl}$ approaches $\mathrm{HO}$ with a non-zero impact parameter relative to the center of mass of $\mathrm{HO}$, then it will have angular momentum and the HO molecule will rotate in such a manner that its rotational angular momentum cancels the angular momentum of the $\mathrm{Cl}$ atom. In the energy interval 1.034 d.u. $\leq E \leq 1.231$ d.u., HO remains a bound molecule and acts dynamically like an anharmonic oscillator that is coupled into the scattering process that occurs between the center of mass of $\mathrm{HO}$ and the $\mathrm{Cl}$ atom.

We next examine how the scattering process changes as we vary the division of the initial HO energy between the rotation and vibration degrees of freedom. We fix the total energy at $E_{\text {tot }}=1.231 \mathrm{~d} . \mathrm{u}$. $=25,000 \mathrm{~cm}^{-1}$ and the sum of the initial rotational and vibrational energies of $\mathrm{HO}$ at value $E_{H O}=0.1969$ d.u. $=4,000 \mathrm{~cm}^{-1}$. Thus, the initial value of $p_{R}$ is held fixed but the incident $\mathrm{Cl}$ can have a range of angular momenta, depending on the initial value of $p_{\theta}$ (in order to keep the total angular momentum of the system fixed at zero). In Figures 3.1 and 3.2, we show the result of varying the fraction of the energy $E_{H O}$ that initially goes to rotation and to vibration of the $\mathrm{HO}$ dimer. In Fig. 3.1, we show scattering dynamics when $p_{\theta}=5.92$ d.u. and vibration has amplitude in the range 1.622 d.u. $\leq r \leq 2.134$ d.u.. In Fig. 3.2, we show scattering dynamics when $p_{\theta}=0.92$ d.u. and vibration has amplitude in the range 1.599 d.u. $\leq r \leq 2.159$ d.u., so the initial energy of $\mathrm{HO}$ is almost entirely 
vibrational. The fractal regions have expanded as the released vibration range increase from 1.622 d.u. $\leq r \leq 2.134$ d.u. to 1.599 d.u. $\leq r \leq 2.159$ d.u.. Because of the stiffness of $\mathrm{HO}$, the regions of discontinuity now cover a significant portion of the initial conditions and have a clear dependence on the initial phase of the $\mathrm{HO}$ vibration.

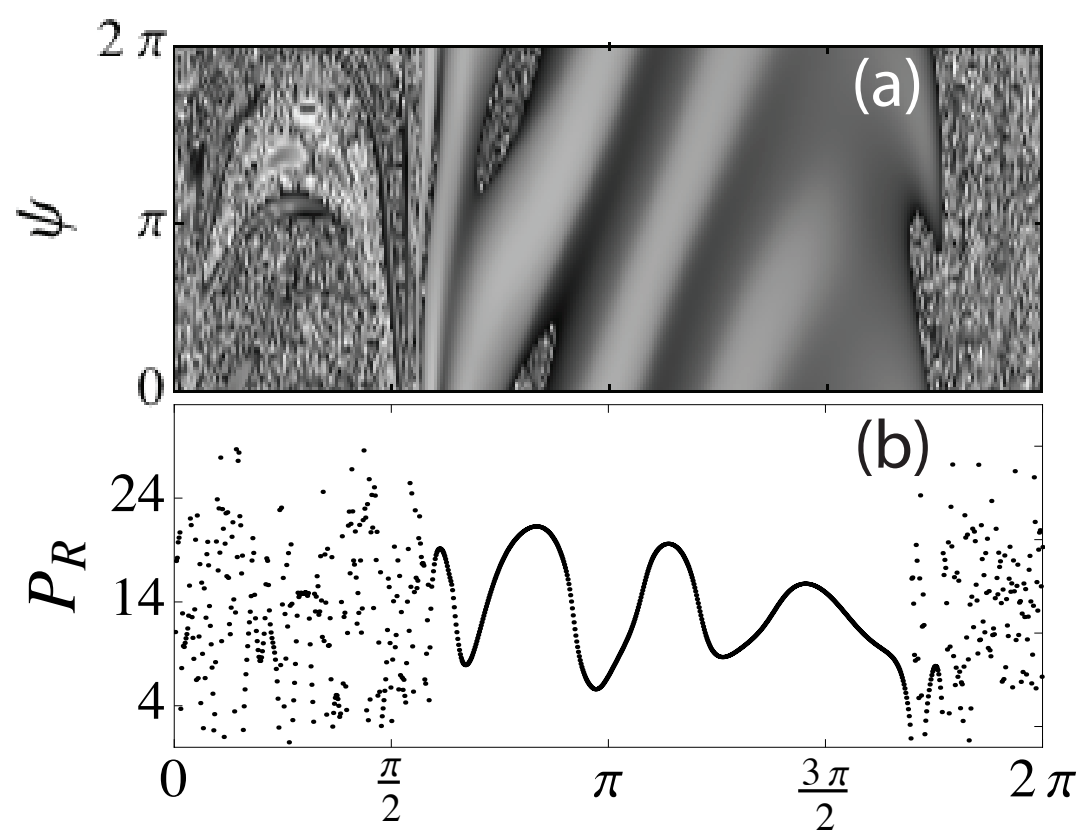

Figure 3.1: The total energy is fixed at $E=1.231$ d.u. $=25,000 \mathrm{~cm}^{-1}$. The energy of the incident chlorine atom is fixed at $E_{C l}=1.034$ d.u. $=21,000 \mathrm{~cm}^{-1}$. The remaining $E_{H O}=0.1969 \mathrm{~d} . \mathrm{u} .=4,000 \mathrm{~cm}^{-1}$ is distributed between rotation and vibration of $\mathrm{HO}$ so that $p_{\theta}=5.92 \mathrm{~d}$.u. and vibration has amplitude in the range 1.622 d.u. $\leq r \leq 2.134$ d.u.. (a) Contour plot of scattered values of $p_{R}$ as a function of $\psi$ and $\chi$. (b) A plot of scattered values of $p_{R}$ as a function of $\chi$ for $\psi=\pi$. The greyscale in (a) ranges from 1 to 32, with 32 the lightest shade. Values of $p_{R}$ at the darkest and lightest points in (a) can be read from (b). ( $p_{R}$ in dimensionless units and $\chi$ in radians.) 


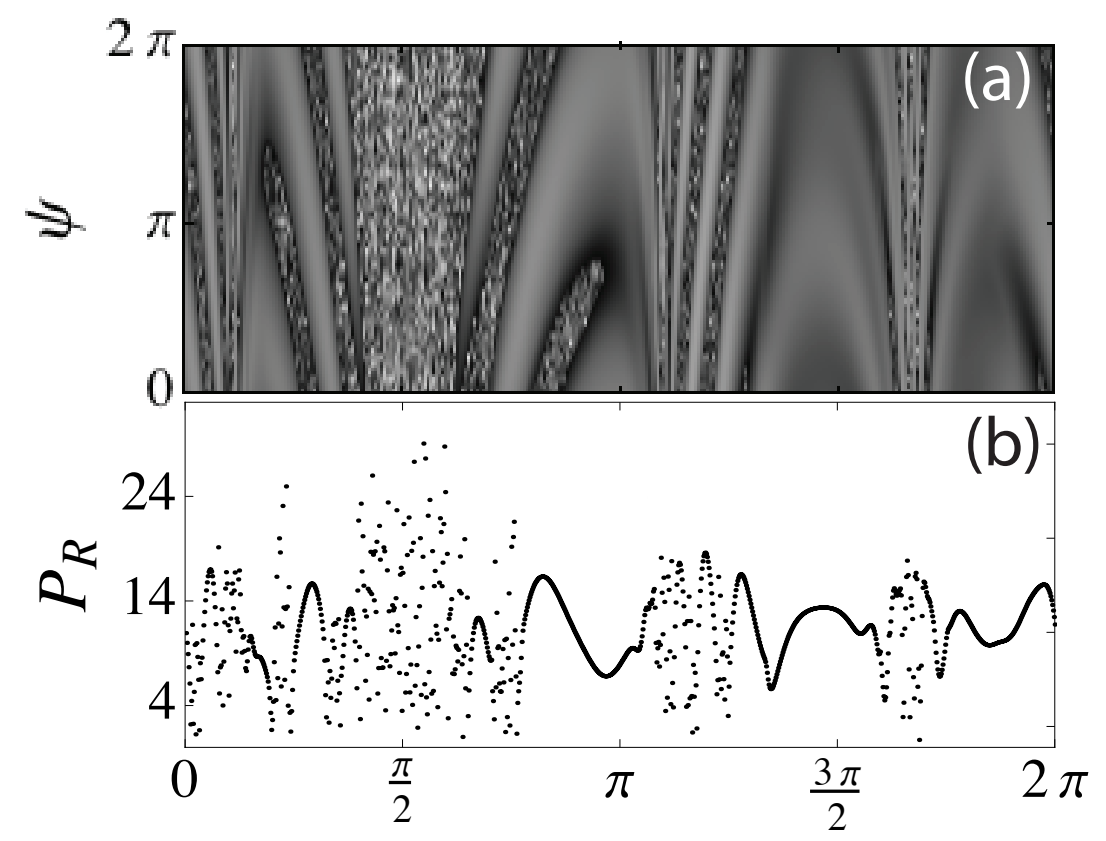

Figure 3.2: The total energy is fixed at $E=1.231 \mathrm{~d} . \mathrm{u} .=25,000 \mathrm{~cm}^{-1}$. The energy of the incident chlorine atom is fixed at $E_{C l}=1.034 \mathrm{~d} . \mathrm{u} .=21,000 \mathrm{~cm}^{-1}$. The remaining $E_{H O}=0.1969$ d.u. $=4,000 \mathrm{~cm}^{-1}$ is distributed between rotation and vibration of $\mathrm{HO}$ so that $p_{\theta}=0.92 \mathrm{~d} . \mathrm{u}$. and vibration has amplitude in the range 1.599 d.u. $\leq r \leq 2.159$ d.u.. (a) Contour plot of scattered values of $p_{R}$ as a function of $\psi$ and $\chi$. (b) A plot of scattered values of $p_{R}$ as a function of $\chi$ for $\psi=\pi$. The greyscale in (a) ranges from 1 to 32, with 32 the lightest shade. Values of $p_{R}$ at the darkest and lightest points in (a) can be read from (b). ( $p_{R}$ in dimensionless units and $\chi$ in radians.) 
The $\mathrm{HO}$ vibration is very stiff compared to that of the $\mathrm{HO}-\mathrm{Cl}$ vibration. This fact has formed the basis of two papers that have modeled the dynamic properties of the $\mathrm{HOCl}$ molecule as a $2 \mathrm{D}$ system, holding the $\mathrm{HO}$ vibration fixed $[14,20]$. To see how well the 2D model holds, we now compare the scattering dynamics of the $2 \mathrm{D}$ and $3 \mathrm{D}$ models of $\mathrm{HOCl}$ at energies above dissociation.
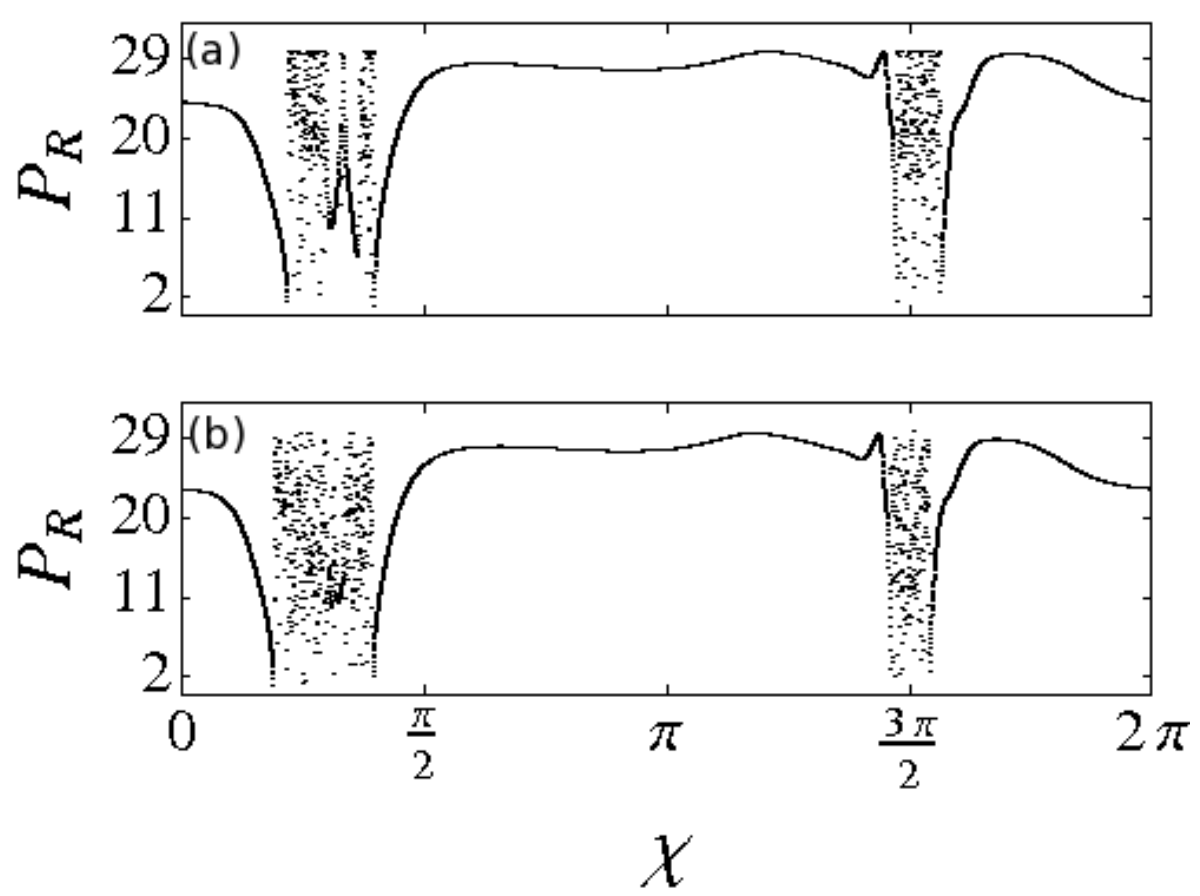

Figure 3.3: (a) 2D scattering of $\mathrm{HOCl}$. (b) 3D scattering of $\mathrm{HOCl}$. Both the $\mathrm{HO}$ of $2 \mathrm{D}$ and $3 \mathrm{D}$ are released slightly excited at the asymptotic region.

In Fig. 3.4, we compare the scattering behavior of $p_{R}$ and $p_{\theta}$ for the 3D case, when the total energy of the $\mathrm{Cl}+\mathrm{HO}$ system is $E=1.231 \mathrm{~d} . u$. $=$ 


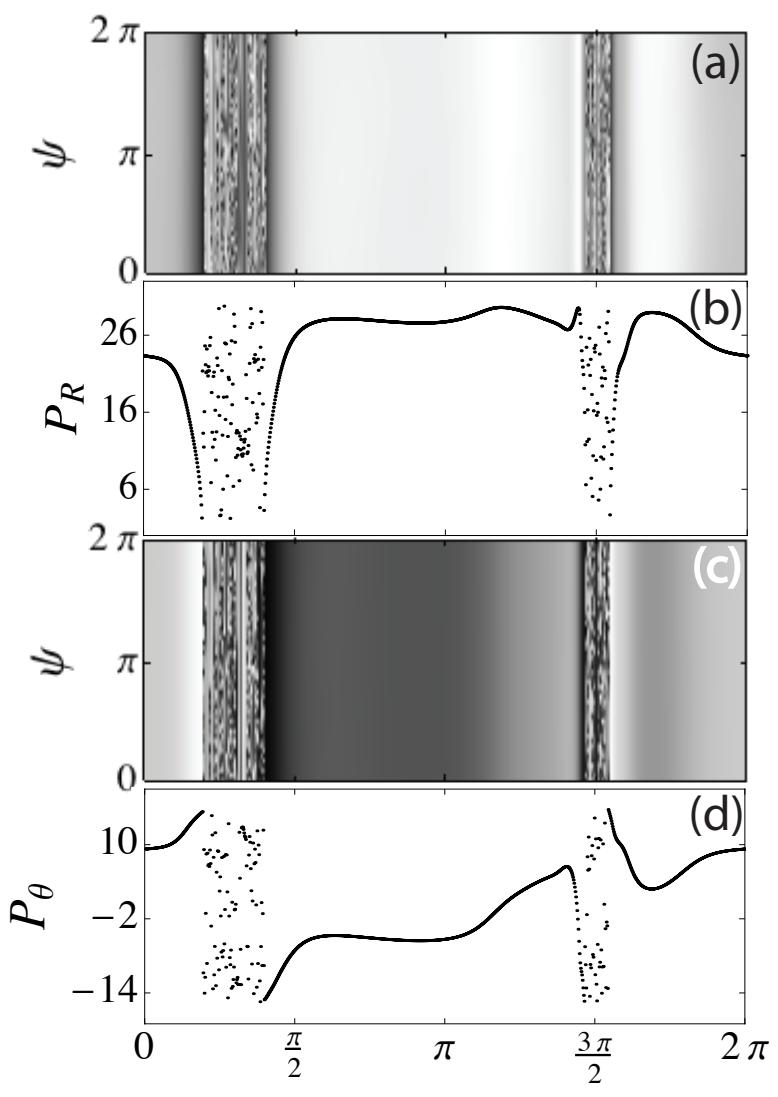

Figure 3.4: The scattering dynamics for the 3D molecule when total energy of the $\mathrm{Cl}+\mathrm{H}-\mathrm{O}$ system is $E=1.231$ d.u. $=25,000 \mathrm{~cm}^{-1}$. The $\mathrm{HO}$ dimer has an initial total energy $E_{H O}=0.03261 .231 \mathrm{~d} . u .=667 \mathrm{~cm}^{-1}$. The initial value of $p_{\theta}$ is $p_{\theta}=5.92$ d.u.. With this value of $p_{\theta}$, $\mathrm{HO}$ is essentially in its vibrational ground state initially. (a) A contour plot of scattered values of $p_{R}$ as a function of $\psi$ (vertical axis) and $\chi$ (horizontal axis). (b) A plot of scattered values of $p_{R}$ as a function of $\chi$ for $\psi=\pi$. Intervals of continuity and chaos are clearly seen. (c) A contour plot of scattered values of $p_{\theta}$ as a function of $\psi$ (vertical axis) and $\chi$ (horizontal axis). (d) A plot of scattered values of $p_{\theta}$ as a function of $\chi$ for $\psi=\pi$. The intervals of continuity and of discontinuity are the same as those of $p_{R}$, indicating that they are an intrinsic property of the molecule. All plots contain 1000 data points along the $\chi$ axis and 50 data points along the $\psi$ axis. The greyscale in (a) and (c) ranges from 1 to 32 , with 32 the lightest shade. ( $p_{R}$ and $p_{\theta}$ in dimensionless units and $\chi$ and $\psi$ in radians.) 
$25,000 \mathrm{~cm}^{-1}$, the $\mathrm{HO}$ molecule has an initial total energy $E_{H O}=0.03282 \mathrm{~d} . \mathrm{u} .=$ $667 \mathrm{~cm}^{-1}$, and an initial angular momentum $p_{\theta}=5.92 \mathrm{~d}$.u. For these parameters, the initial energy of $\mathrm{HO}$ is almost entirely rotational. The initial range of oscillation of $\mathrm{HO}$ is 1.8333 d.u. $\leq r \leq 1.8446$ d.u., so $\mathrm{HO}$ is close to its minimum vibration energy. Figs. 3.4.a and 3.4.c show contour plots of scattered values of $p_{R}$ and $p_{\theta}$, respectively, as a function of $\psi$ (vertical axis) and $\chi$ (horizontal axis). Figs. 3.4.b and 3.4.d show plots of scattered values of $p_{R}$ and $p_{\theta}$, respectively as a function of $\chi$ for fixed $\psi=\pi$ (for $\psi=\pi$, HO is initially at the turning point with minimum value of $r$ ). All of the plots in Fig. 3.4 contain 1000 data points for $\chi$ and 50 data points for $\psi$. Figs. 3.4.b and 3.4.d show that the scattering data contain large ranges of $\chi$ with intervals of continuity, and two shorter ranges of $\chi$ that contain a fractal distribution (of measure zero) of singularities. These are actually points of intersection of the stable manifold of the outer NHIM with the set of initial conditions. The fractal set of singularities form the boundaries of a complimentary fractal set of intervals of continuity, so the scattering function becomes discontinuous in this region. In Fig. 3.4, these intervals of continuity and singular points appear to be approximately independent of the $\psi$ (the initial configuration of $\mathrm{HO}$ ), indicating that the scattering dynamics is well approximated by the 2D model used in $[14,20]$ in which the $\mathrm{HO}$ vibration is held fixed. The fractal nature of the scattering process is intrinsic to the $\mathrm{Cl}-\mathrm{HO}$ system above dissociation and same fractal structure will appear in all scattering functions. This can be seen in the Figs. 3.4.a and 3.4.c, which show asymptotic of $p_{R}$ and $p_{\theta}$ after 
the scattering process. The fractal structure of this plots is identical. Further discussions between 2D and 3D HOCl can be found in Appendix B.

The qualitative behavior of the scattering dynamics of the $\mathrm{HOCl}$ molecule, just above dissociation, depends critically on the initial excitation energy of the $\mathrm{HO}$ dimer. If the dimer is initially close to its ground state, the scattering dynamics has only small regions of initial conditions that show fractal behavior and the scattering dynamics can be well described by the $2 \mathrm{D}$ model. If the HO dimer is initially in a fairly high excited state, a large portion of initial conditions show fractal behavior. In this case, it would appear that the scattering dynamics can no longer be approximated by the 2D model. 


\section{Chapter 4}

\section{Periodic Orbits of $\mathrm{HOCl}$}

The dynamics of $\mathrm{HOCl}$, just above dissociation, is dominated by the $\mathrm{Cl}-\mathrm{HO}$ interaction because $\mathrm{Cl}$ can dissociate, while $\mathrm{HO}$ remains bound. In Fig. 2.2, we show the potential energy surface, $V\left(R, \theta, r_{e q}\right)$, for the molecule with HO fixed at its equilibrium value $r=r_{e q}=1.85 \mathrm{~d} . u$. The potential energy surface has a symmetry line at $\theta=0$, and this symmetry line contains a saddle at $R=R_{s d}=3.16$ d.u. and energy $E_{s d}=0.840$ d.u. $=17,053 \mathrm{~cm}^{-1}$, which is well below the dissociation energy. For energies below $E_{s d}$, the $\mathrm{Cl}$ atom is confined to one side $(\theta>0)$ or the other $(\theta<0)$ of the molecule. For energies above this saddle, the $\mathrm{Cl}$ atom can oscillate back and forth over the saddle. As we shall see, the symmetry line $\theta=0$ plays an important role in the classical and quantum dynamics of $\mathrm{HOCl}$.

The saddle at $\left(\theta=0, R=R_{s d}=3.16\right.$ d.u. $)$ is a source of chaos in both the 2D and 3D models of $\mathrm{HOCl}$. In the 2D model of $\mathrm{HOCl}[14,20]$, the HO complex is held fixed at its equilibrium configuration $r=r_{e q}$, and it is possible to visualize the phase space dynamics using surfaces of section (SOS). In the $2 \mathrm{D}$ system, above dissociation, the global dynamics is dominated by two unstable (hyperbolic) fixed points, one belongs to a periodic orbit oscil- 
lating over the saddle point of the potential and the other lies far out in the asymptotic region [14]. Both fixed points are the source of stable and unstable manifolds that, above dissociation, impart the classical Cl-HO scattering dynamics a fractal structure [18]. The unstable (stable) manifolds, as they evolve forward (backward) in time, form a fractal network of tendrils that define the chaotic region of the phase space (see Appendix A). The stable and unstable manifolds from both fixed points enter the same regions of the phase space and become "synchronized". This synchronization is caused by the existence of heteroclinic connections between unstable periodic orbits. Together, the stable and unstable manifolds, from both fixed points, define the fractal structure of all dynamical functions that characterize this region of the phase space.

Evidence of this synchronization of the stable and unstable manifolds can be seen in the 2D system using Poincaré Surfaces of Section (SOS). In Figs. 4.1.a and 4.1.b, we plot $p_{R}$ versus $R$ each time the trajectory passes the point $\theta_{e q}$ (the position of the absolute minimum potential energy) when $p_{\theta}>0$, for energies $E=21,000 \mathrm{~cm}^{-1}$ and $E=25,000 \mathrm{~cm}^{-1}$, respectively. The initial conditions are taken from the neighborhood of the stable manifold of the hyperbolic fixed point in the asymptotic region. The SOS shows the structure of the large chaotic sea that exists above dissociation. Some of the tendrils of the stable manifold are visible in these plots. Almost all trajectories in this large chaotic sea (above dissociation) are scattering trajectories and will eventually disappear into the asymptotic region, although the time scale for 

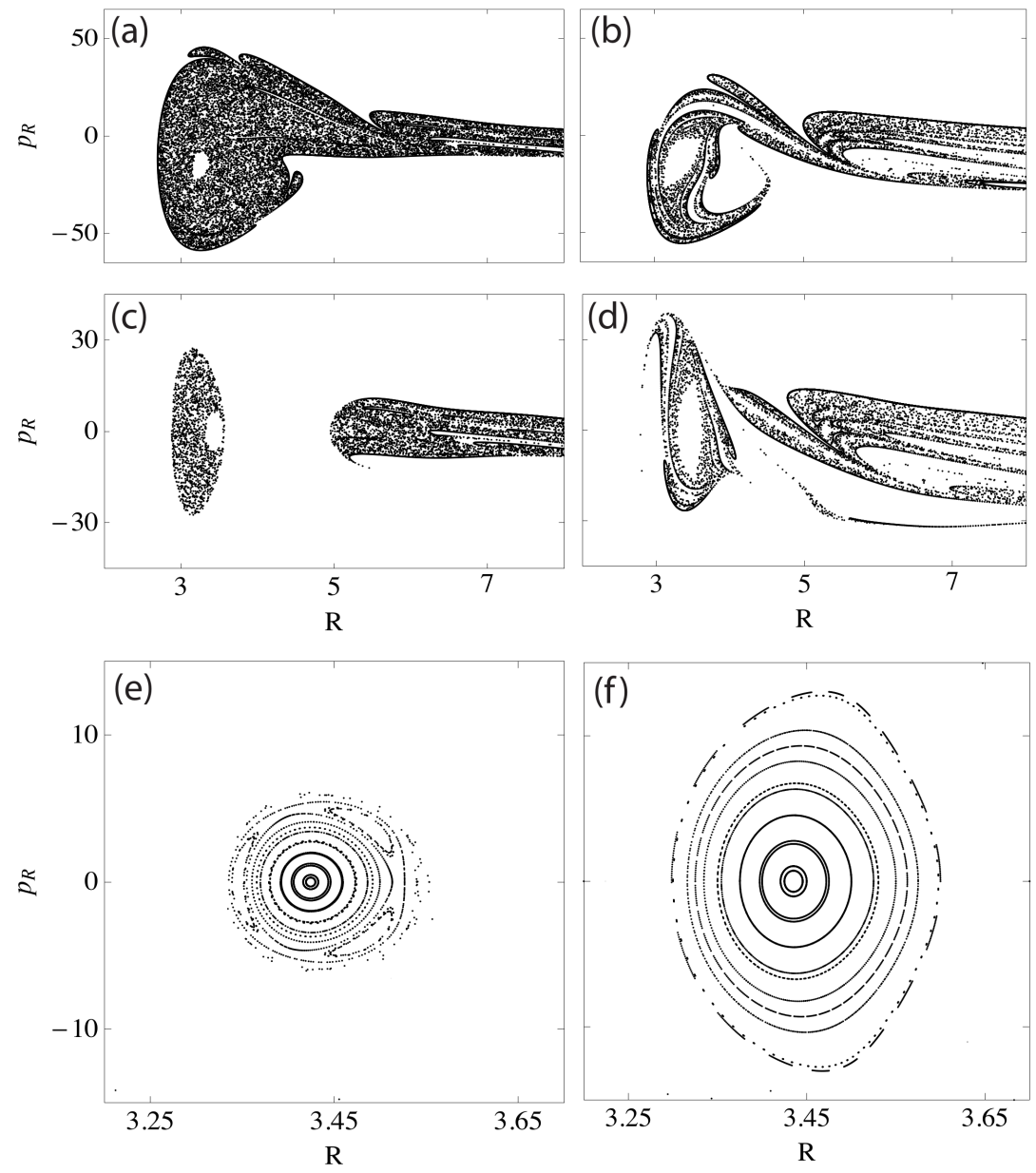

Figure 4.1: Surfaces of section for 2D HOCl. (a) $p_{R}$ versus $R$ when $\theta=$ $\theta_{e q}=1.34 \mathrm{rad}$ for $E=1.03 \mathrm{~d} . u$. $=21,000 \mathrm{~cm}^{-1}$. (b) $p_{R}$ versus $R$ when $\theta=\theta_{e q}$ for $E=1.23 \mathrm{~d}$.u. $=25,000 \mathrm{~cm}^{-1}$. (c) $p_{R}$ versus $R$ when $\theta=0$ for $E=1.03$ d.u. $=21,000 \mathrm{~cm}^{-1}$. (d) $p_{R}$ versus $R$ when $\theta=0$ for $E=1.23$ d.u. $=$ $25,000 \mathrm{~cm}^{-1}$. (e) $p_{R}$ versus $R$ when $\theta=0$ for $E=1.03 \mathrm{~d} . u$. $=21,000 \mathrm{~cm}^{-1}$ in the neighborhood of the $B_{0}$ periodic orbit. (f) $p_{R}$ versus $R$ when $\theta=0$ for $E=1.23$ d.u. $=25,000 \mathrm{~cm}^{-1}$ in the neighborhood of the $B_{0}$ periodic orbit. 
this to happen varies with the particular trajectory and can approach infinity.

One remarkable feature of the plots in Figs. 4.1.a and 4.1.b is the large island of KAM tori that is embedded in the chaotic sea. This large island of quasi periodic orbits in the SOS shows the existence of a stable periodic orbit that exists above dissociation. It corresponds to a stable periodic oscillation of the $\mathrm{Cl}$ atom about the end of the $\mathrm{O}$ atom. It was seen in [14] and identified as $B_{0}$. In Figs. 4.1.c and 4.1.d, we plot a SOS for the same trajectories, but now we plot $p_{R}$ versus $R$ every time the trajectories pass through $\theta=0$. The chaotic sea extends throughout the phase space. In Figs. 4.1.e and 4.1.f, we show the structure of the stable island surrounding the periodic orbit $B_{0}$, for the SOS take at $\theta=0$, for energies $E=1.03$ d.u. $=21,000 \mathrm{~cm}^{-1}$ and $E=1.23$ d.u. $=25,000 \mathrm{~cm}^{-1}$. The path of this periodic orbit is shown in Fig. 2.2 as the dashed line that runs across the saddle at $R=3.44 \mathrm{~d} . u$.

For the 3D HOCl dynamics, surfaces of section can be constructed, but they would involve plots of $\left(p_{R}, R, p_{r}, r\right)$, each time $\theta=\theta_{e q}$ or $\theta=0$, and therefore cannot be visualized easily because they are four dimensional surfaces. However, it is possible to visualize some global features of the system. A large chaotic sea continues to exist above dissociation, and we have found that a stable periodic orbit, with some similarities to the $2 \mathrm{D} B_{0}$ periodic orbit, exists in the $3 \mathrm{D}$ system. The $3 \mathrm{D} B_{0}$ stable periodic orbit, and its unstable "sister" 3D $B_{0}$ orbit, emerge as the result of a saddle-center bifurcation at energy $E=23,990 \mathrm{~cm}^{-1}=1.18 \mathrm{~d} . u$. and have period $\tau=130 \mathrm{~d} . u$. (Fig. 4.2). The stable $3 \mathrm{D} B_{0}$ orbit survives up to energy $E \approx 28,200 \mathrm{~cm}^{-1}$, where 
it has period $\tau \approx 140 d . u$, and the unstable 3D $B_{0}$ orbit survives up to energy $E \approx 30,000 \mathrm{~cm}^{-1}$. A search of the phase space did not reveal any other stable periodic orbits in this energy range near the InM.

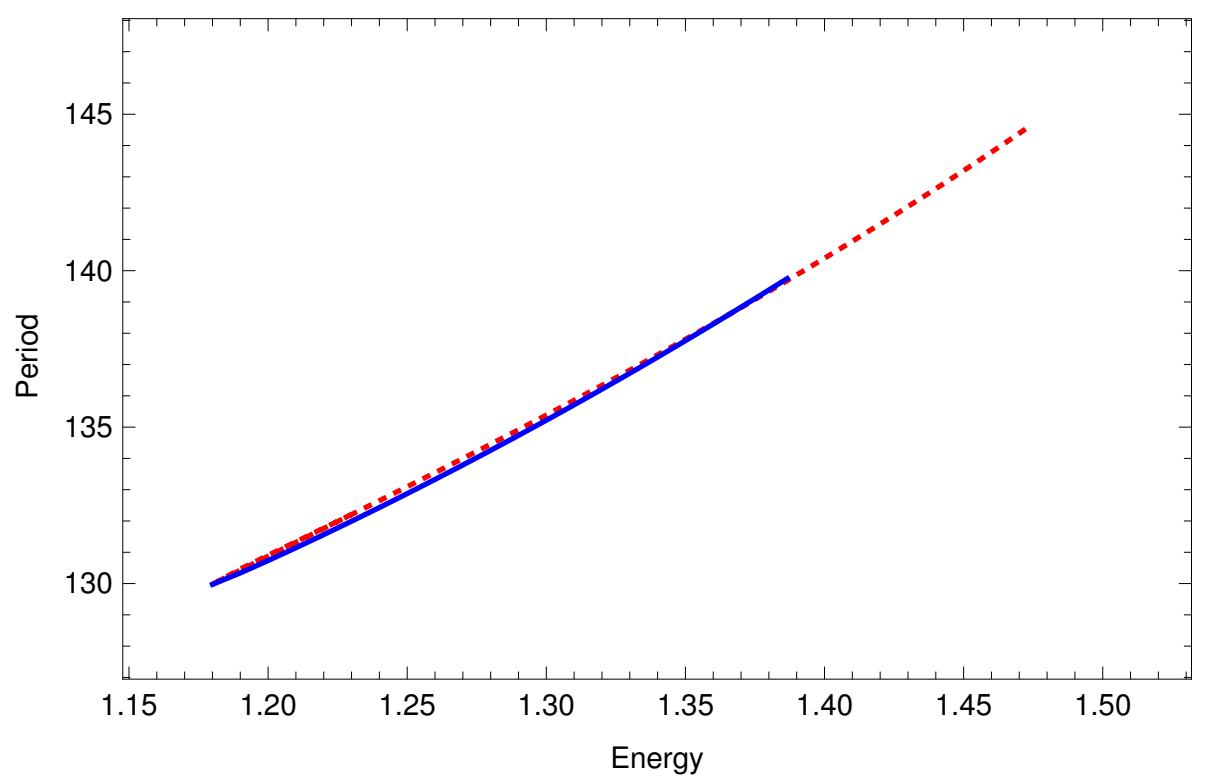

Figure 4.2: Bifurcation diagram of 3D $B_{0}$ stable (solid) and unstable (dashed). Both the period and energy are in the dimensionless units.

In Fig. 4.3.a, (Fig. 4.3.b) we show the path of the HOCL molecule, in the $x^{\prime}-z^{\prime}$ plane, when it moves along the $2 \mathrm{D} B_{0}$ (3D stable and unstable $\left.B_{0}\right)$ orbits. In all cases, the periodic motion of the molecule largely involves the $\mathrm{H}$ atom oscillating off the end of the $\mathrm{O}$ atom, with small compensating movements of $\mathrm{O}$ and $\mathrm{Cl}$ to ensure that the total angular momentum of the molecule is zero. In Fig. 4.4.a, we show a projection of the dynamics of the stable and unstable 3D periodic orbits (which we will call the 3D $B_{0}$ orbits) onto the $R-\theta$ plane, for energy $E=1.23 d \cdot u .=25,000 \mathrm{~cm}^{-1}$ ), and we show 
the path of the 2D $B_{0}$ in the same figure. They are similar, in that they lie in the same region of the $R-\theta$ plane, although the $3 \mathrm{D} B_{0}$ orbit has oscillatory behavior due to its involvement with the HO internal dynamics.

There are also some very important differences between the 3D and 2D $B_{0}$ periodic orbits. The $2 \mathrm{D} B_{0}$ periodic orbit crosses the $\theta=0$ line in the $2 \mathrm{D}$ phase space and the $\mathrm{Cl}$ atom passes back and forth around the $\mathrm{O}$ atom. The 3D $B_{0}$ periodic orbits do not cross the $3 \mathrm{D} \theta=0$ surface, but each consists of two mirror image periodic orbits, one to the left and the other to the right of the $\theta=0$ surface. Classically the $2 \mathrm{D} B_{0}$ orbit is a $(1,2)$ resonance of the $(\theta, R)$ motions ( $\theta$ oscillates through one period each time $R$ oscillates through two periods). The $3 \mathrm{D} B_{o}$ periodic orbits involve a $(1,1,3)$ resonance of the $(\theta, R, r)$ motions. However, this motion occurs for the orbit on the left and the right sides of the $\theta=0$ surface, so if they could be combined, the $(\theta, R)$ part of the motion would be the same as for the 2D $B_{o}$ orbit. Plots of $R(t)$, $\theta(t)$, and $r(t)$, for one complete oscillation of the $3 \mathrm{D} B_{0}$ periodic orbit, are shown in Figs. 4.4.c, 4.4.e, and 4.4.g, respectively.

Short periodic orbits, such as the $2 \mathrm{D}$ and $3 \mathrm{D} B_{0}$ periodic orbits serve as organizing centers for large regions of the dynamics, and play an important role in the vibrational quantum dynamics of the molecule. References $[5,6]$ showed that such periodic orbits in $2 \mathrm{D} \mathrm{HOCl}$ form the backbone of bound states and quasibound states of the molecule. The wave function sets up standing waves along such paths. Above dissociation, the quasibound states are long lived states of the $\mathrm{HOCl}$ complex that can significantly affect reaction 

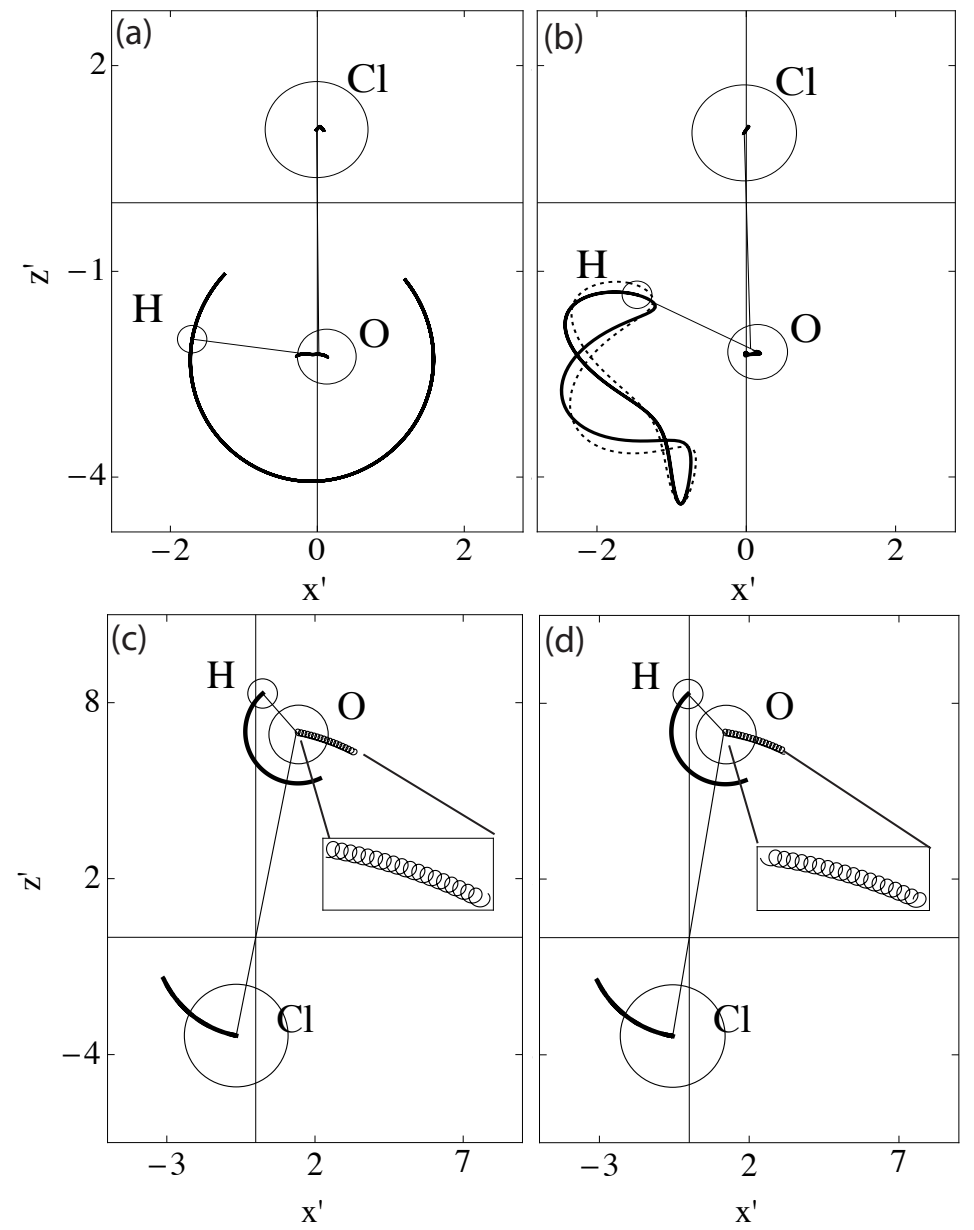

Figure 4.3: Sketch of path covered by periodic orbits in $x^{\prime}-z^{\prime}$ plane. (a) The $2 \mathrm{D}$ orbit $B_{0}$. (b) The stable 3D $B_{0}$ orbit (solid) and the unstable 3D B0 orbit (dotted). (c) The $2 \mathrm{D}$ orbit $B_{1}$. (d) The $3 \mathrm{D}$ orbit $B_{1}$. Figures (c) and (d) look similar because the $\mathrm{HO}$ vibration is barely excited for the $3 \mathrm{D}$ orbit. 


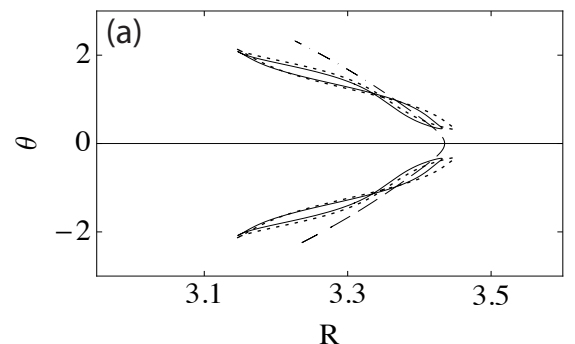

(b)
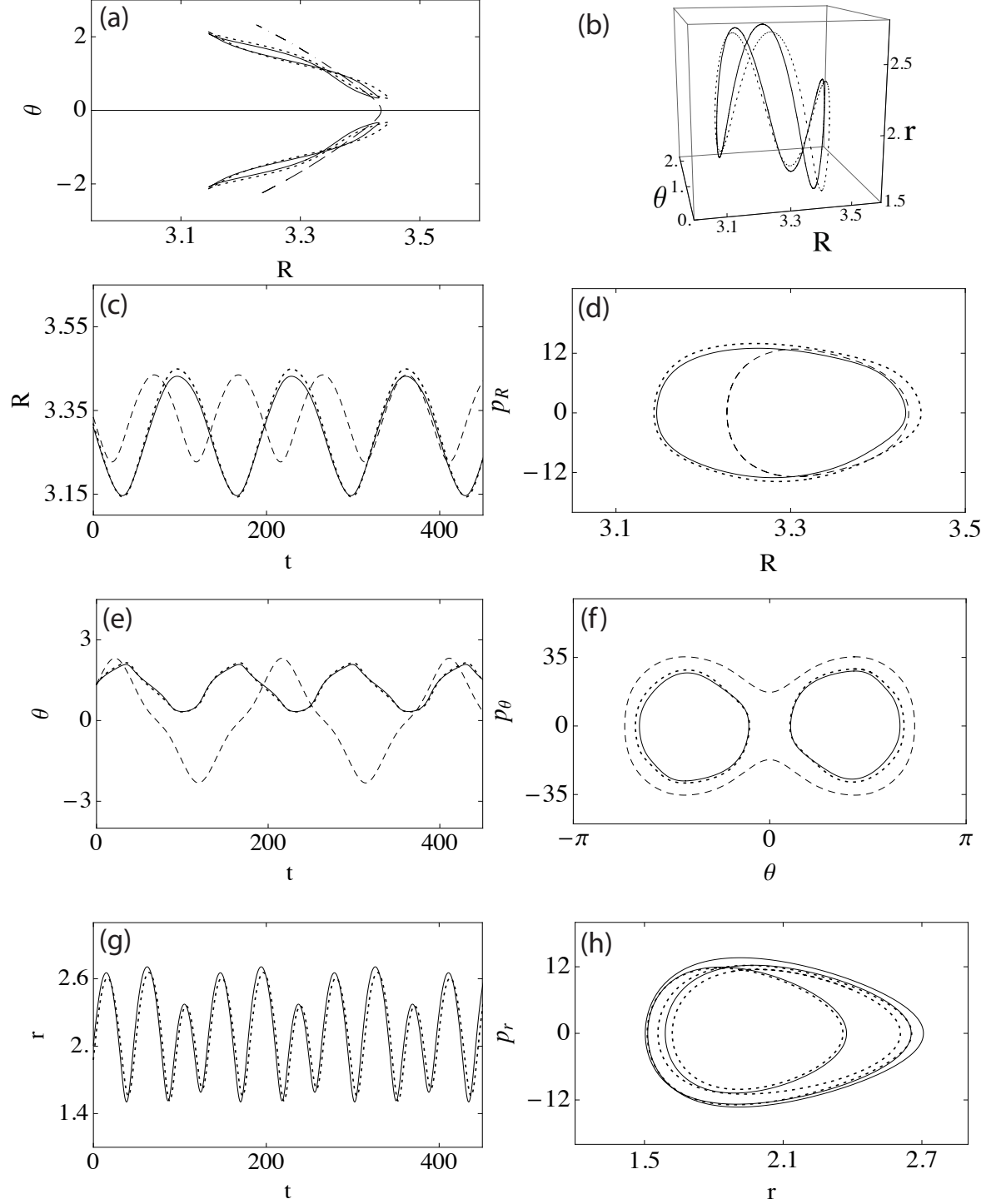

Figure 4.4: Projected images of the $2 \mathrm{D} B_{0}$ (dashed), the stable $3 \mathrm{D} B_{0}$ (solid) periodic orbit, and the unstable $3 \mathrm{D}$ B0 orbit (dotted). (a) The $(R, \theta)$ plane. (b) The $(R, \theta, r)$ surface (3D orbits only). (c) The $R(t)$ versus $t$. (d) The $\left(p_{R}, R\right)$ plane. (e) The $\theta(t)$ versus $t$. (f) The $\left(p_{\theta}, \theta\right)$ plane. $(\mathrm{g})$ The $r(t)$ versus $t$ (3D $B_{0}$ only). (h) The $\left(p_{r}, r\right)$ plane (3D $B_{0}$ only). 
rates [16,21-23]. In Figs. 4.4.d, 4.4.f, and 4.4.h, we plot projections of the stable and unstable 3D $B_{0}$ motion in the $\left(p_{R}, R\right),\left(p_{\theta}, \theta\right)$, and $\left(p_{r}, r\right)$ planes. These figures allow us to use semi-classical quantization arguments to estimate the energy spacing of longitudinal quantum states that can "live" on the $B_{0}$ periodic orbit, at least for the case when transverse states on the orbit are not excited. The area enclosed by the orbits in these figures is equal to the classical actions $J_{R}=\oint p_{R} d R, J_{\theta}=\oint p_{\theta} d \theta$, and $J_{r}=\oint p_{r} d r$. In dimensionless units, the actions are $\frac{J_{\theta}}{\hbar} \approx 75.4, \frac{J_{R}}{\hbar} \approx 6.3$, and $\frac{J_{r}}{\hbar} \approx 58.5$, so the action of the $3 \mathrm{D}$ $B_{0}$ orbit at energy $E=1.23$ d.u. is $S \approx 140.2$ d.u.

We can use the above information about the $3 \mathrm{D} B_{0}$ orbit to make some predictions about the quantum behavior of the orbit. We have found that the stable 3D periodic orbit $B_{0}$ exists over the whole energy interval $E \approx 1.18$ d.u. to $E \approx 1.40 \mathrm{~d} . u$. and the period of the orbit varies linearly from $\tau \approx 130 \mathrm{~d} . u$. at energy $E=1.18$ d.u. to $\tau \approx 139$ d.u. at energy $E=1.38$ d.u. and obeys the relation $\tau(E) \approx 130+45.5(E-1.18)$ in d.u. The relation between the period of the orbit $\tau$, the energy $E$, and the action $S(E)$ is $\tau=\frac{d S(E)}{d E}$ [7]. Therefore the action varies as $S(E) \approx 12.1+76.3 E+22.7 E^{2}$ (in d.u.) over the energy range for which the stable 3D $B_{0}$ orbit exists (Fig. 4.5). The semi-classical quantization conditions specify that the action is quantized in units of Planck's constant $h$. Each time the energy of the orbit increases by $\Delta E \approx 0.0074$ d.u., that action changes by $\Delta S \approx 2 \pi d . u$., and in this interval there must be one value of $E$ that fulfills the semi-classical quantization condition [24]. In fact, for $\mathrm{HOCl}$ there will be two quantum states in this energy interval because each 
3D $B_{0}$ orbit has a mirror image. We expect that the two identical stable 3D $B_{0}$ orbits will give rise to symmetric and antisymmetric quantum states that live on both sides of the InM.

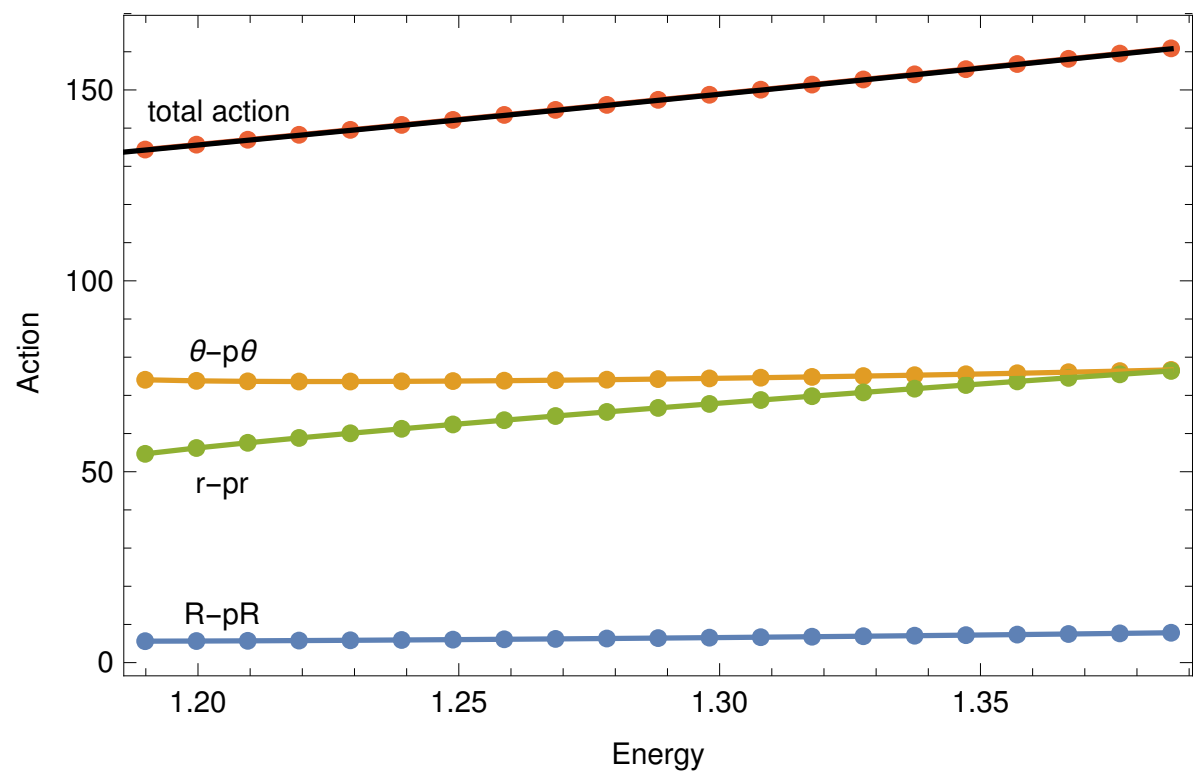

Figure 4.5: Action of 3D $B_{0}$ (stable) orbit. The black solid line is the action derived from the relation of period and energy. Both the action and energy are in dimensionless units. 


\section{Chapter 5}

\section{The Invariant Manifold at $\theta=0, p_{\theta}=0$}

Besides conserving energy and angular momentum, $\mathrm{HOCl}$ has another fundamental symmetry. Its Hamiltonian is an even function of both $\theta$ and $p_{\theta}$. This means that the Hamiltonian is invariant under reflection through the point $\theta=0$, and it implies that the derivatives of $H$ with respect to $\theta$ and $p_{\theta}$ are both identically zero. Trajectories with initial conditions, $\left(p_{\theta}=\right.$ $0, \theta=0)$ will retain those values of $\left(p_{\theta}, \theta\right)$ in all their subsequent motion. They therefore move on a 2D Invariant Manifold (InM) for which energy is conserved and only the coordinates $\left(p_{R}, R, p_{r}, r\right)$ can vary. This corresponds to a linear configuration of the molecule, one for which the $\mathrm{Cl}, \mathrm{O}$, and $\mathrm{H}$ lie along a line, in that order. The $\mathrm{HO}$ complex can vibrate and the $\mathrm{Cl}$ can dissociate along that line.

The dynamics on the InM has two degrees of freedom and, therefore, we can visualize the dynamics on the InM using SOSs. In Fig. 5.1.a, we plot the potential energy $V(R, r, \theta=0)$ on the InM for $2.75 \leq R \leq 4.2$ and $1.45 \leq r \leq 2.3$. The InM has a potential energy minimum at $(R=3.15, r=1.80)$ and a saddle point at $(R=4.03, r=1.84)$. The dark line in Fig. 5.1.a is a line of

potential energy minima (a minimum energy pathway (MEP)). We can show 

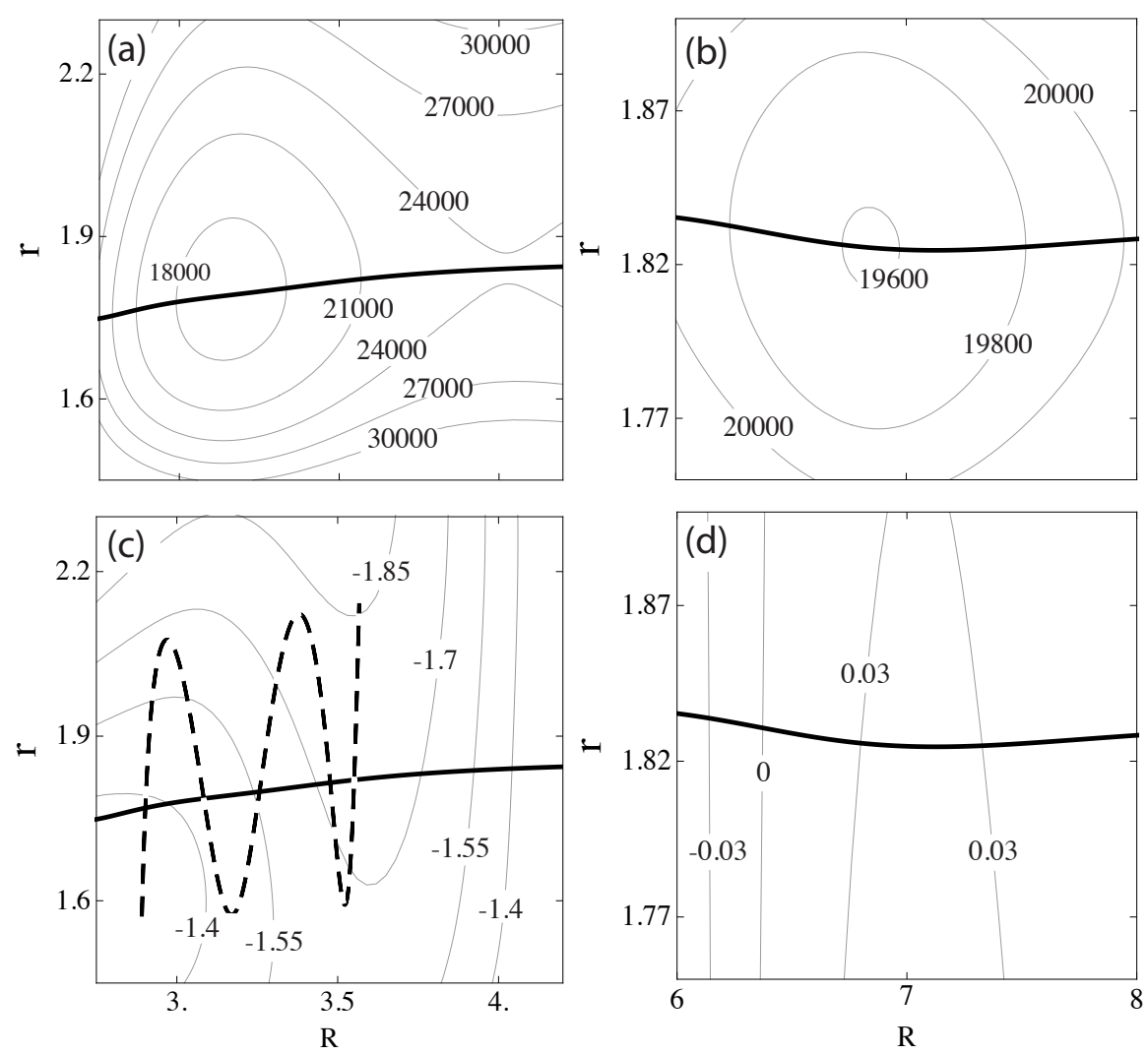

Figure 5.1: The potential energy and normal stability of the Invariant Manifold. The dark solid line in all figures is the MEP. (a) The potential energy $V_{I M}(R, r)$ for 2.75 d.u. $\leq R \leq 4.2$ d.u. and 1.45 d.u. $\leq r \leq 2.3$ d.u.. Contour lines are lines of constant potential energy. (b) The potential energy $V_{I M}(R, r)$ for 6.0 d.u. $\leq R \leq 8$ d.u. and 1.75 d.u. $\leq r \leq 1.90$ d.u.. Contour lines are lines of constant potential energy. (c) The variation of $\kappa$ as a function of $R$ and $r$ for $2.75 d . u . \leq R \leq 4.2 d . u$. and $1.45 d . u . \leq r \leq 2.3 d . u$.. In this region $-1.85 d . u . \leq \kappa \leq-1.40$ d.u. and the InM is transversally unstable. The dashed line is the large period-five periodic orbit (see Fig. 5.2.a), with energy $E=1.23$ d.u. $=25,000 \mathrm{~cm}^{-1}$, on the InM. Contour lines are lines of constant $\kappa$. (d) The variation of $\kappa$ as a function of $R$ and $r$ for 6.0 d.u. $\leq R \leq 8.0$ d.u. and 1.75 d.u. $\leq r \leq 1.9$ d.u. . In this region -0.03 d.u. $\leq \kappa \leq 0.03$ d.u. and the InM is weakly stable. Contour lines are lines of constant $\kappa$. In all these plots, the dark solid line is the MEP. 
the dynamics on the InM using a SOS, with the intersection condition along the MEP. Such a SOS requires the use of Birkhoff coordinates $p_{R_{B}}, R_{B}$, where $R_{B}$ indicates distance along the MEP and $p_{R_{B}}$ indicates the parallel component of the momentum along the MEP.

Surfaces of section showing $\left(p_{R_{B}}, R_{B}\right)$, each time $r$ crosses the MEP are shown in Fig. 5.2 for energies; (a) $E=25,000 \mathrm{~cm}^{-1}$, (b) $E=30,000 \mathrm{~cm}^{-1}$;, and (c) $E=35,000 \mathrm{~cm}^{-1}$. We see that for energy $E=25,000 \mathrm{~cm}^{-1}$ the dynamics on the InM is very stable. It contains a dominant stable periodic orbit at $R_{B}=0.57(R=3.17)$ and a saddle point at $R_{B}=1.43(R=4.03)$ . The invariant manifolds of the saddle point in this SOS form a thin chaotic strip that surrounds a large island of KAM tori. As energy increases, new resonances emerge in the neighborhood of the stable periodic orbit and the chaotic strip widens. We can estimate the number of quantum states on the InM. For example, if we consider the period five orbit (chain of five islands) in Fig. 5.2.a and plot the projection of the orbit onto $p_{R}, R$, and $p_{r}, r$, surfaces, then semiclassical arguments indicate that this periodic orbit could support a number of quantum states. Thus, motion on the InM is potentially significant for the quantum dynamics of the molecule.

The mechanism for dissociation of the molecule, when its dynamics is confined to the InM, has interesting behavior. For the case when the initial condition of the molecule lies in the chaotic region of the SOS (near the separatrix), the rate of dissociation has echoes. Trajectories cycle around the region near the chaotic layer in Fig. 5.2.a and after each revolution, a fraction of 

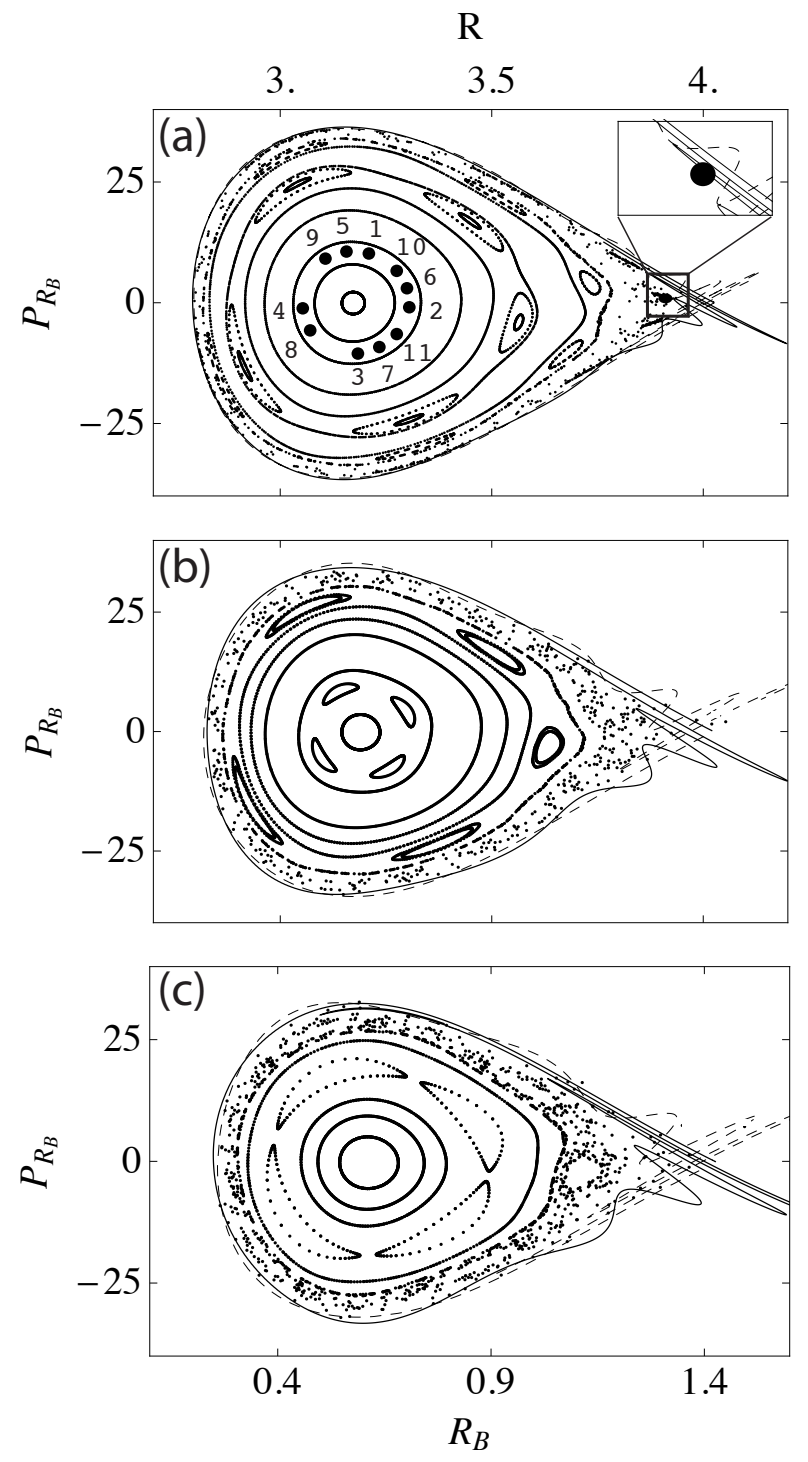

Figure 5.2: Surfaces of Section of Birkhoff coordinates $\left(p_{R_{B}}, R_{B}\right)$ along the MEP on the Invariant Manifold each time a trajectory crossing the MEP in Fig. 5.1. These figures show numerous periodic orbits and a saddle point on the Invariant Manifold. In all three cases, the stable and unstable manifolds, associated with the saddle point, and the chaotic layer associated with them are plotted. (a) $E=1.23$ d.u. $=25,000 \mathrm{~cm}^{-1}$. The eleven black dots near the center show an effective SOS for the trajectory in Fig. 5.5 for time interval $200 \leq t \leq 600$. The inset shows the initial condition for echoes (see Fig. 5.3). (b) $E=1.48$ d.u. $=30,000 \mathrm{~cm}^{-1}$. (c) $E=1.72$ d.u. $=35,000 \mathrm{~cm}^{-1}$. 
the trajectories are ejected into the asymptotic region. This periodic emission of trajectories has been predicted for microwave waveguides and nanometer scale electron waveguides [25-27], and has been observed experimentally in microwave cavities[28] The period of the echoes (about 180 d.u.) gives a measure of the time needed to complete one revolution, along the chaotic strip, around the large KAM island in Fig. 5.2.a. The trajectories are caught up in the fractal structure of tangles in the chaotic region $[14,18]$, and cycle around the chaotic layer until they eventually escape into the asymptotic region as the molecule dissociates. In Fig. 5.3, we show the flux, $P$, of escaping trajectories (measured at $R=7 d . u$. as a function of time. The period $\tau_{f x}$ of the flux pulses is determined by the time it takes a trajectory to cycle around the chaotic layer in Fig. 5.2.a (about $\tau_{f x}=180$ d.u.) $[25,27]$. The period of such echoes has also been related to the development parameter [25] of the stable and unstable manifolds of the InM saddle point.

\subsection{Stability of the Invariant Manifold}

We can determine the stability of the InM by linearizing the equations of motion about $\theta=0$ and $p_{\theta}=0$. The part of the Hamiltonian that depends on $\theta$ and $p_{\theta}$, in this neighborhood, reduces to

$$
H \approx \frac{p_{\theta}^{2}}{2 \delta_{1} R^{2}}+\frac{p_{\theta}^{2}}{2 \delta_{2} r^{2}}+\frac{1}{2} \kappa \theta^{2}+\ldots
$$

where $\delta_{1}=1930$ and $\delta_{2}=159$. Thus, in the neighborhood of the InM, the potential energy is either a harmonic oscillator or an inverted oscillator, 

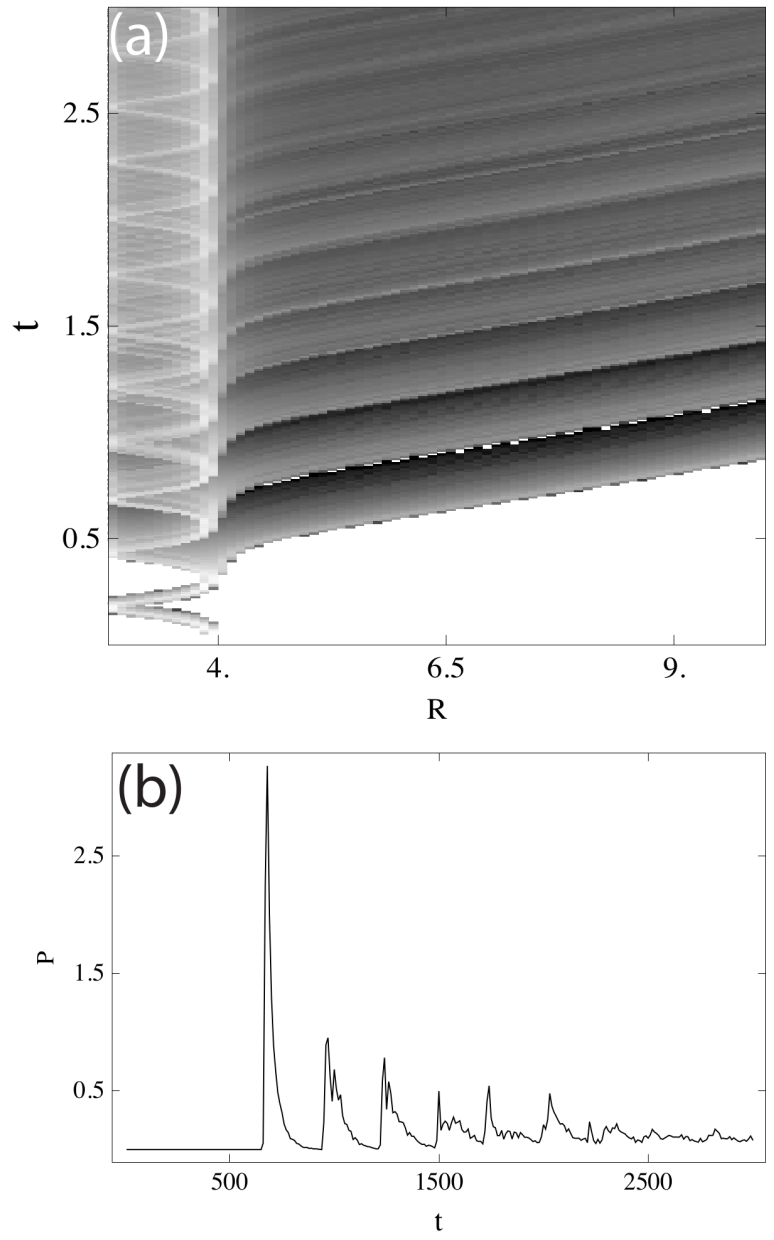

Figure 5.3: (a) The density plot of 100,000 escaping trajectoris initially released in the inset of Fig. 5.2.a ( $\mathrm{t}$ in units of 1000 d.u.). (b) The flux, $P$ (in units of 1000 d.u.), of escaping trajectories (measured at $R=7.0$ d.u.) as a function of time $t$, shows echoes, as trajectories escape to the asymptotic region. The echoes have a period of about $180 \mathrm{~d} . \mathrm{u}$. 
depending on the sign of $\kappa$. The value of $\kappa$ is a function of $R$ and $r$. In Fig. 5.1.c, we show the variation of $\kappa$ for a range of values $2.75 \leq R \leq 4.2$ and $1.45 \leq r \leq 2.3$. In this region $-1.85 \leq \kappa \leq-1.4$. so the potential energy has the form of an inverted oscillator and motion in the neighborhood of the InM is hyperbolic. If this normal instability is larger than the tangential instability of the dynamics on the InM, then the InM is called a Normally Hyperbolic Invariant Manifold (NHIM) [8]. This type of InM has been studied in the literature for their potential importance in determining reaction rates of molecules [29,30]. In Fig. 5.1.d, we show the variation of $\kappa$ for $6.0 \leq R \leq 8.0$ and $1.75 \leq r \leq 1.90$. The InM becomes weakly stable in this region of the phase space. Below we consider these two regions of the InM separately.

\subsubsection{Unstable InM}

When the direction transverse to the InM (the $\theta$ direction) is unstable, it can only support quasibound quantum states. The lifetime of a quantum state on the unstable InM is the same as that of a collection of classical orbits distributed about the InM with the same probability density as the quantum state. In both cases, the lifetime is determined by the eigenvalues of the stable and unstable manifolds normal to the InM. To illustrate this, let us consider the point on the InM with $R=3.2$ and $r=r_{e q}=1.8$. For these values of $R$ and $r$, the Hamiltonian in the neighborhood of the InM can be written (see Eq. 5.1)

$$
H \approx \frac{p_{\theta}^{2}}{2 \delta_{a v}}-\frac{1}{2}|\kappa| \theta^{2}+\ldots
$$


where $\delta_{a v}^{-1}=\left(\delta_{1} R_{s d}^{2}\right)^{-1}+\left(\delta_{2} r_{e q}^{2}\right)^{-1}$ so that $\delta_{a v}=502.5$. A normal mode analysis of the stable (unstable) manifold of the fixed point at $\left(\theta=0, p_{\theta}=0\right)$, shows that the approach (escape) rate for the $\operatorname{InM}$ is $\omega(-\omega)$, where $\omega=\sqrt{\frac{|\kappa|}{\delta_{a v}}}=0.082$. The slope of the unstable manifold, in the $p_{\theta}, \theta$ plane is $\frac{\Delta p_{\theta}}{\Delta \theta}=41$. This large change in $p_{\theta}$ for a small change in $\theta$ is due to the large inertia of the $\mathrm{Cl}$ atom.
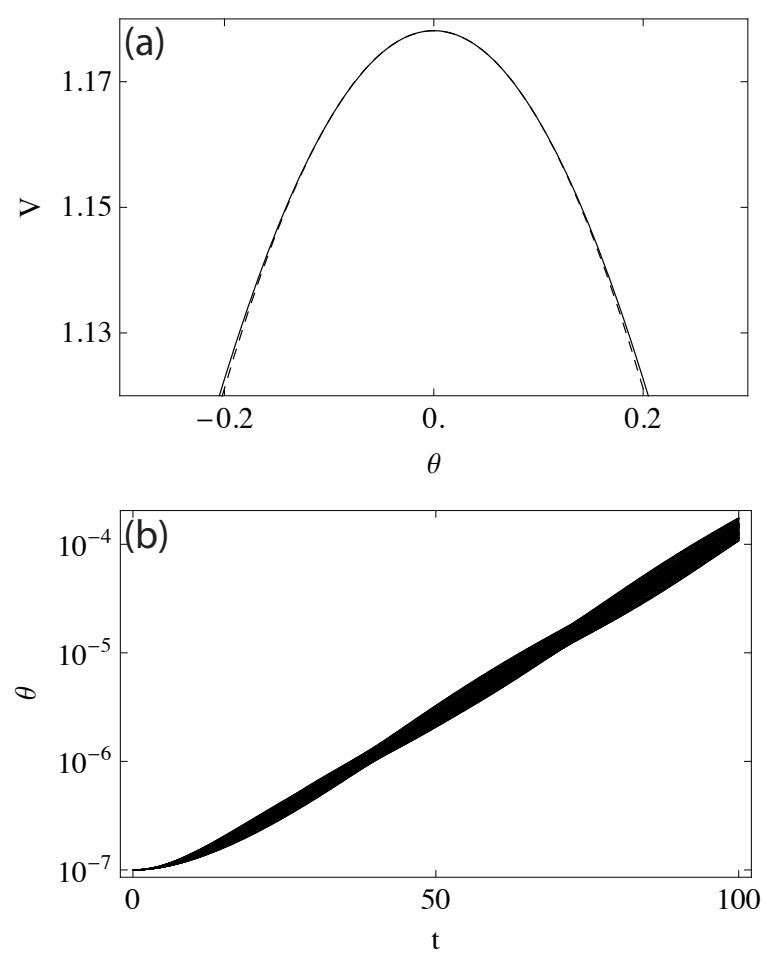

Figure 5.4: (a) The potential energy normal to the InM at $R=4.025 d$.u. and $r=r_{e q}=1.85 d . u$. In this region the flow normal to the InM is hyperbolic, with stable and unstable manifolds normal to the InM. (b) Plot of $\theta$ versus $t$ for 100 initial conditions of the orbits with initial conditions taken a distance $\Delta \theta=10^{-7}$ from the large period-five orbit in Fig. 5.2.a. The slope of $\log (\theta)$ is $\omega=0.072$.

The decay rate $\omega$ of quantum states on the unstable InM can also be 
determined numerically from the behavior of trajectories in the neighborhood of the InM. In Fig. 5.4.a, we plot the potential energy normal to the InM at $R=4.025 \mathrm{~d} . u$. and $r=r_{e q}=1.85 \mathrm{~d} . u$.. In this region, the potential energy is an inverted oscillator and the flow normal to the InM is hyperbolic, so the InM is unstable. In Fig. 5.4.b, we plot $\theta$ versus $t$ for 100 initial conditions taking orbits with initial conditions a distance $\Delta \theta=10^{-7}$ from the large period-five island chain in Fig. 5.2.a. The slope of $\log (\theta)$ is $\omega=0.072$, very close to the theoretical estimate.

The length of time that a classical trajectory remains in the neighborhood of the InM (has $p_{\theta}$ and $\theta$ close to zero), if it starts near the InM, depends on its distance of closest approach to the InM. In Fig. 5.5, we show the evolution of $R, \theta$, and $r$, as a function of time, for a trajectory that starts with initial values of $R$ and $r$ very close to one of the numbered black dots near the center of Fig. 5.2.a. These two trajectories lie on opposite sides of the stable manifold of the unstable InM and have different long-time behavior. We show projections onto the coordinate axes for two orbits with energy $E=1.23$ d.u. $=25,000 \mathrm{~cm}^{-1}$ close to the stable manifold of the unstable InM in the neighborhood of the ring of numbered black dots in Fig. 5.2.a. One trajectory always has $\theta<0$ and the other trajectory passes from $\theta<0$ to $\theta>0$.

The quantum behavior provides less room to maneuver. Semiclassical arguments indicate that the InM can hold a number of quantum states. However the lifetime of those states is determined by the lifetime of a state on 

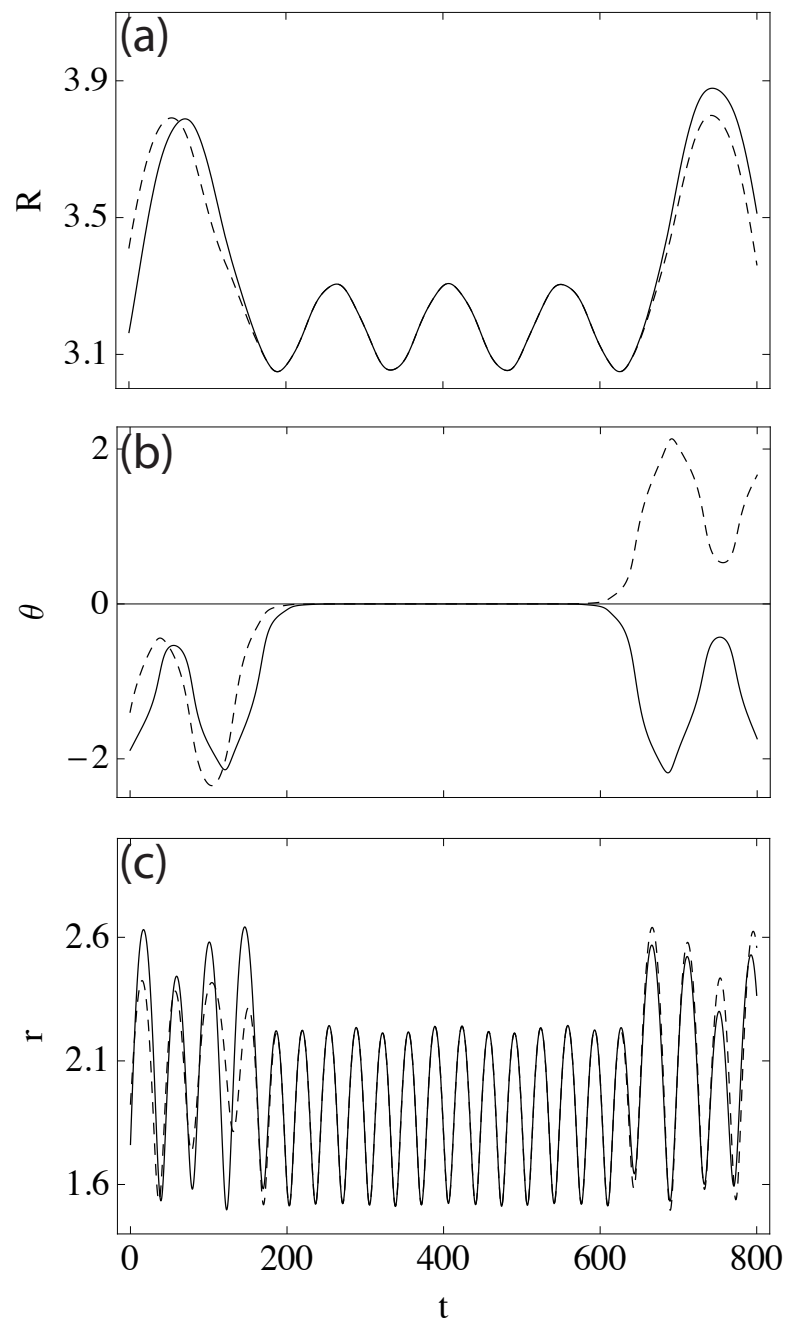

Figure 5.5: Projections onto the coordinate axes for two orbits with energy $E=1.23 \mathrm{~d} . u .=25,000 \mathrm{~cm}^{-1}$ close to the stable manifold of the unstable InM in the neighborhood of the ring of numbered black dots in Fig. 5.2.a. The trajectories have different long time behavior. (a) $R(t)$ versus $t$. (b) $\theta(t)$ versus $t$. (c) $r(t)$ versus $t$. 
the inverted oscillator in the $\theta$ direction [31]. If we assume that the initial state in the $\theta$ direction is the minimum uncertainty wave packet $\psi(\theta, 0)=$ $\left(\frac{\delta \omega}{\pi}\right)^{1 / 4} e^{-\frac{1}{2} \delta \omega \theta^{2}}$, then the state at time $t$ is [32],

$$
\psi(\theta, t)=\frac{\sqrt{-i \operatorname{csch}(\omega t)} \exp \left(\frac{i \delta \omega \theta^{2} \operatorname{coth}(\omega t)}{2}-\frac{\delta \omega \theta^{2} \operatorname{csch}^{2}(\omega t)}{4\left(\frac{1}{2}-\frac{1}{2} i \operatorname{coth}(\omega t)\right)}\right)}{\sqrt{2} \sqrt[4]{\pi} \sqrt[4]{\frac{1}{\delta \omega}} \sqrt{\frac{1}{2}-\frac{1}{2} i \operatorname{coth}(\omega t)}} .
$$

For this state, most of the initial probability is centered at $\left(p_{\theta}=0, \theta=0\right)$ with a width of about $\delta p_{\theta}= \pm 10$ and $\delta \theta= \pm 0.2$. It takes a time of about $t=15 \mathrm{~d} . u$. for most of the initial probability to decay out of the neighborhood of the unstable InM. This rapid decay of the wave packet off of the InM indicates that echoes likely cannot be seen in the dissociation of the $\mathrm{HOCl}$ molecule.

\subsubsection{Stable InM}

In Fig. 5.1.b, we show the potential energy surface on the InM in the region where it is transversally stable. There is a potential energy local minimum that lies slightly below the dissociation energy, and there is a periodic orbit on the InM, but its action is not large enough to hold a quantum state.

The fact that this region of the InM is marginally stable led us to search for another classically significant periodic orbit in this region of the phase space. We found both a 2D periodic orbit and a 3D periodic orbit, which we call the 2D $B_{1}$ and $3 \mathrm{D} B_{1}$, periodic orbits, respectively. The 2D $B_{1}$ orbit is located at $R \approx 11$, and corresponds to an libration of $\mathrm{H}$ about $\mathrm{O}$ 
and slight oscillations of $\mathrm{O}$ and $\mathrm{Cl}$, in opposite directions, in such a way as to maintain zero total angular momentum of the molecule. However, the action associated with each degree of freedom in this motion is not large enough to hold a quantum state. Therefore, $B_{1}$ is classically significant, but likely can't be excited in the $\mathrm{HOCl}$ molecule. 


\section{Chapter 6}

\section{Conclusions}

In the Appendix A [15], we first demonstrate the tools used to analyze scattering from an inverted Gaussian potential in an oscillating field (one and half degrees of freedom). There are two unstable fixed points located at positve and negative infinity along the x-axis. On the SOS, flow of conserved area is illustrated to show the concept of Liouville's theorem. We choose heteroclinic intersections and their tendrils for the boundaries of conserved areas and label the hierarchy of the areas (tangles) as a function of the mapping time. The structure of the flow demonstrates the idea of fractal scattering. To specify the rates of transmition and reflection, we further build up a dynamical tree, the transfer matrix, and determine its topological entropy.

The vibrational motion of small molecules, such as $\mathrm{HOCl}$, provides a laboratory for studying the quantum-classical correspondence (QCC) in coupled nonlinear oscillator systems. Using a 3D energy surface which governs the vibrational dynamics of $\mathrm{HOCl}$ below and just above dissociation [16], we have analyzed the vibrational dynamics of $\mathrm{HOCl}$ for a subspace of the molecular

dynamics for which total angular momentum is zero $[18,19]$. We show that the $2 \mathrm{D} \mathrm{HOCl}$ is a good approximation to the $3 \mathrm{D} \mathrm{HOCl}$ when the $\mathrm{HO}$ vibration 
is only slightly excited at the asymptotic region. The fractal dimensions of 3D and $2 \mathrm{D}$ can be found in Appendix $\mathrm{B}$ which uses a high resolution data run on the machines on Texas Advanced Computing Center (TACC). The intrinsic scattering property of $3 \mathrm{D} \mathrm{HOCl}$ is imbedded in higher dimensional manifolds and their intersections as illustrated in a simplier version in Appendix A.

In order to apply the concept of Liouville's theorem, in higher dimension we focus on a 2D Invariant Manifold which is imbedded in the 3D phase space. The dynamics of $\mathrm{HOCl}$ is dominated by this Invariant Manifold which is transversally unstable for small spacing between $\mathrm{Cl}$ and $\mathrm{HO}$ complex (a NHIM), and stable for large spacing. We focus on the Invariant Manifold as the major tool for further investigation because of its $2 \mathrm{D}$ property. In the NHIM region of the Invariant Manifold, there is a dominant saddle point. We use homoclinic tendrils from the saddle point and their intersections to identify the flow on the InM. We use Birkhoff coordinate to obtain a clear SOS and find several stable islands set around the chaotic layer that leads to echoes on the InM. In addition, we have identified the $3 \mathrm{D} B_{0}$ orbits that emerge as the result of a saddle-center bifurcation at energy $E=23,990 \mathrm{~cm}^{-1}=1.18$ d.u. The action associated to the $3 \mathrm{D} B_{0}$ is sufficient to support a large quasibound state in $\mathrm{HOCl}$. The InM separates two mirror image $B_{0}$ periodic orbits each of which can hold a large number of quantum states. These periodic orbits have the capability of forming significant quasibound states of the molecule above dissociation. 
Appendices 


\section{Appendix A}

\section{Fractal scattering in a radiation field}

\section{A.1 Introduction}

Chaos can profoundly affect the scattering dynamics of classical systems and it can exert significant influence on the quantum analog of the scattering dynamics. A monochromatic radiation field coupled to the nonlinear dynamics of an otherwise field-free scattering process gives rise to an extremely rich scattering dynamics governed by a fractal set of singularities that is embedded in all dynamical functions involved with the scattering process. This fractal set of singularities occurs when the incoming particle asymptotes intersect the tangles of the invariant manifolds in the asymptotic region. The recipe for extracting information about chaotic scattering processes and the underlying

fractal structure that governs that dynamics has been developed by Jung and others for a variety of model systems [9-13,33]. The method described here for obtaining the underlying fractal structure for chaotic scattering process can also be applied to scattering processes in the absence of a radiation field, as has been shown in [14] where the chaotic scattering dynamics of a molecular system is analysed. More general reviews of scattering chaos can be found in $[34,35]$. 
A particularly fruitful system for studying fractal scattering dynamics, both classically [11-13] and quantum mechanically [36,37], is the negative chlorine ion $\mathrm{Cl}^{-}$in the presence of a radiation field. In [11-13], the fractal structure of the scattering dynamics for this system was studied in great detail. However, one important physical aspect was not addressed - namely, how to determine the fraction of trajectories that is transmitted through the scattering region versus the fraction that is reflected from the scattering region. That is the issue we address in this Appendix.

We begin in Sect. A.2 with a discussion of properties of the Hamiltonian that we use to study the fractal scattering process. In Sect. A.3, we define a "fundamental region" using invariant manifolds of the driven potential and we analyze the fractal network of tendrils formed by these invariant manifolds. In Sect. A.4, we describe the symbolic dynamics that is used to develop a branching tree that reflects the complexity of the network of tendriles in the asymptotic region of the phase space. We also define a transfer matrix for the branching treee and use it to give an estimate of the fraction of orbits that are reflected and transmitted for a given incident direction. Finally in Sect. A.5, we make some concluding remarks.

\section{A.2 The Hamiltonian}

The model system we consider is that of an electron of mass $m$ and charge $q$, in the presence of a monochromatic radiation field whose electric field $E$ is linearly polarized along the direction of motion of the electron. The 
electron, while being driven by the radiation, scatters from a one-dimensional inverted Gaussian atomic potential. The Hamiltonian can be written

$$
H_{1}\left(p^{\prime}, x^{\prime}, t\right)=\frac{\left(p^{\prime}\right)^{2}}{2 m}-V_{0} e^{-\left(x^{\prime} / \delta\right)^{2}}-x^{\prime} F \sin (\omega t)
$$

where $p^{\prime}$ and $x^{\prime}$ are the momentum and displacement of the electron in the lab frame and $F=q E$. We can transform from the lab frame $\left\{p^{\prime}, x^{\prime}\right\}$ to a reference frame $\{p, x\}$ moving with the electron (the Kramers-Henneberger frame $(\mathrm{KH})[38,39])$ via a canonical transformation whose generating function is $\mathcal{F}_{3}\left(p^{\prime}, x, t\right)=-p^{\prime} x+\frac{F}{\omega}\left[-x \cos (\omega t)+\frac{p^{\prime}}{m \omega} \sin (\omega t)\right]$ [40]. Then $p=-\frac{\partial \mathcal{F}_{3}}{\partial x}$ and $x^{\prime}=-\frac{\partial \mathcal{F}_{3}}{\partial p^{\prime}}$ and the transformed Hamiltonian $H_{2}$ is given by $H_{2}=H_{1}+\frac{\partial \mathcal{F}_{3}}{\partial t}$. We can further transform this time-periodic Hamiltonian to a time-independent Hamiltonian with coordinates $\{p, x, J, \phi\}$ by letting $\phi=\omega t$ and writing

$$
H_{3}(p, x, J, \phi)=\frac{p^{2}}{2 m}+V_{0} e^{-(x-\alpha(\phi) / \delta)^{2}}+G(\phi)+\omega J
$$

where $\alpha(t)=\alpha_{0} \sin (\phi), \alpha_{0}=F / \omega^{2}$, and $G(\phi)=\frac{F^{2}}{2 m \omega^{2}}\left(2 \sin (\phi)^{2}-\cos (\phi)^{2}\right)$. The action variable $J$ for the radiation field evolves independently of the motion of the electron, and $\dot{\phi}=\omega$ at all times. In the KH-frame, the inverted Gaussian potential oscillates back and forth and, in the asymptotic region, the electron motion is free motion since $\dot{p}=0$ and $\dot{x}=p$. The Hamiltonian is invariant under the generalized symmetry transformation $x \rightarrow-x$ and $\phi \rightarrow \phi+\pi$, but not under the transformation $x \rightarrow-x$ alone. Therefore, there is an asymmetry in the scattering dynamics of this system. As we will see below, the fraction of incident particles that are transmitted and reflected will differ depending on the incident direction. 
In subsequent sections, we use atomic units so the electron mass $m=1$ a.u. and we choose $V_{0}=0.27035$ a.u., $\delta=2$ a.u., $\omega=0.65$ a.u.and $\alpha_{0}=0.9$ a.u. which have been shown to describe the quantum behaviour of a negative chlorine ion $\mathrm{Cl}^{-}$in the presence of a laser field [11,41-43].

\section{A.3 Fractal Scattering}

The fractal structure of the scattering process can be seen in a Poincaré surface of section (PSS) of the dynamics. We solve Hamilton's equations in the KH-frame and plot $p$ and $x$ each time $\phi=\pi / 2+n 2 \pi$, where integer $n=$ $0,1,2, \ldots$ The inverted Gaussian system, in the absence of radiation, has three primary period- $2 \pi$ periodic orbits (fixed points of the PSS): a stable fixed point at $(p=0, x=0)$, an unstable periodic orbit $\left(F_{L}\right)$ at $(p=0, x=-\infty)$, and an unstable periodic orbit $\left(F_{R}\right)$ at $(p=0, x=+\infty)$. We define a fundamental region $R$, in the PSS, whose boundaries are given by segments of the invariant manifolds of the outer fixed points of the system. The invariant manifolds and the fundamental area $R$ are shown in Fig. A.1.a. The invariant manifolds are located by taking a line of initial points with fixed $x$ but varying $p>0$ near $p=0$ and in the neigborhood of the separatrix in the absence of radiation. We evolve the points in time and determine which points escape and which points do not escape. The point separating these two behaviors is on the invariant manifold. This process is repeated for different values of $x$ until a segment of the manifold is mapped out. The curves $n-u$ and $s-n$ are segments of the unstable and stable manifolds of $F_{L}$, respectively. The curves $\mathrm{r}-\mathrm{p}$ and $\mathrm{p}-\mathrm{v}$ 

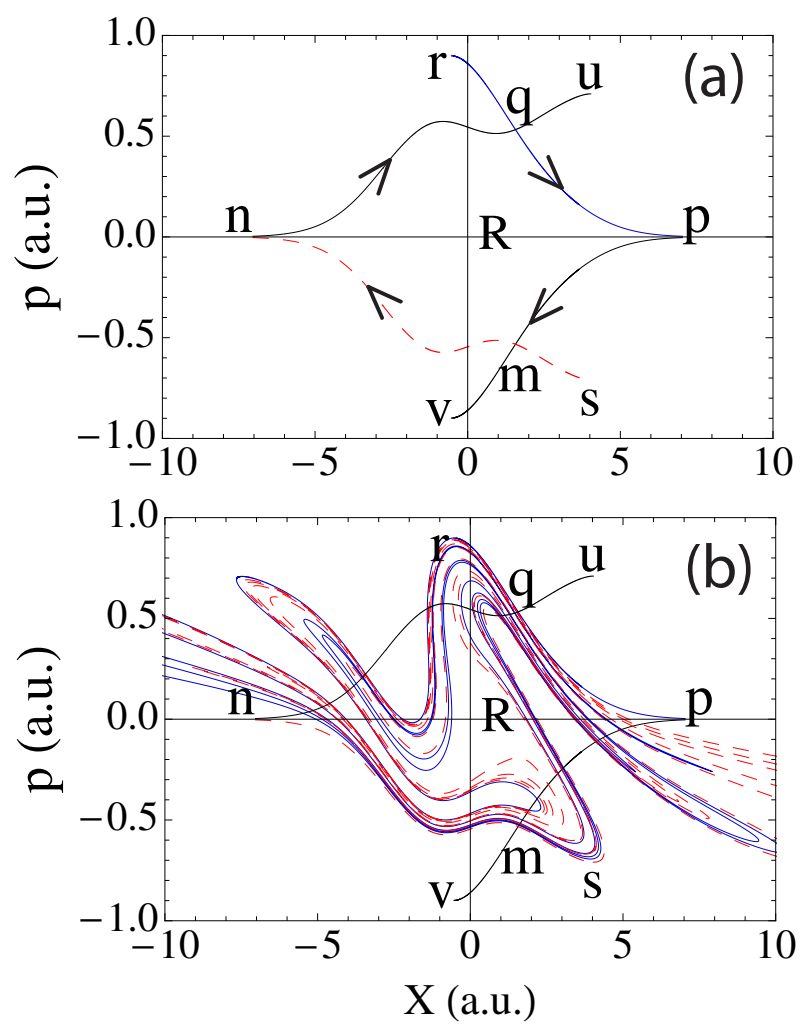

Figure A.1: A Poincaré surface of section with $p$ and $x$ plotted each time $\phi=\pi / 2$. (a) The curves n-u and s-n ( r-p and p-v) are segments of the unstable and stable manifolds of $F_{L}\left(F_{R}\right)$, respectively. The fundamental region is formed by the curves n-q, q-p, n-m and m-p. (b) The segments of the stable manifolds r-p (blue-solid line) and n-s (red dashed line) are integrated backward in time for four iterations of the PSS. 
are segments of the stable and unstable manifolds of $F_{R}$, respectively. The fundamental area is defined by the curves n-q, q-p, n-m and m-p. Because the invariant manifolds approach the $p=0$ axis exponentially as $x \rightarrow \pm \infty$, it is sufficient to consider the segments shown in the figure.

In Fig. A.1.b, we show four iterations backward in time of the segments r-p and s-n of the stable manifolds. The stable manifolds cannot intersect themselves and they cannot intersect each other. However, they can intersect the unstable manifolds. The curves, as they are iterated backward in time, must enclose the same area enclosed in Fig. A.1.a. These constraints cause them to form a fractal network of tendrils as $t \rightarrow-\infty$.

We can distinguish points associated with the asymptotic region from those associated with the fundamental region. This becomes clear in Figs. A.2.a and A.2.b, where the shaded regions with positive indices contain points that lie outside the fundamental region. Iterations of these shaded regions are shown for times ranging from $t=-5$ to $t=+4$ (nine iterations of the PSS). As the map is iterated backward in time, the shaded regions form more and more complex filaments that cut across the fundamental region. As the shaded regions are iterated forward in time, they approach their respective asymptotic regions. In Fig. A.2.a, we follow the point $\alpha_{n}(-5 \leq n \leq 4)$ which is incident from the right at time $n=-5$, but happens initially to be inside one of the tendrils in the asymptotic region at that time. In Fig. A.2.b, we follow the point $\beta_{n}(-5 \leq n \leq 4)$ which is incident from the left at time $n=-5$ and is initially inside a tendril in the asymptotic region at that time. 

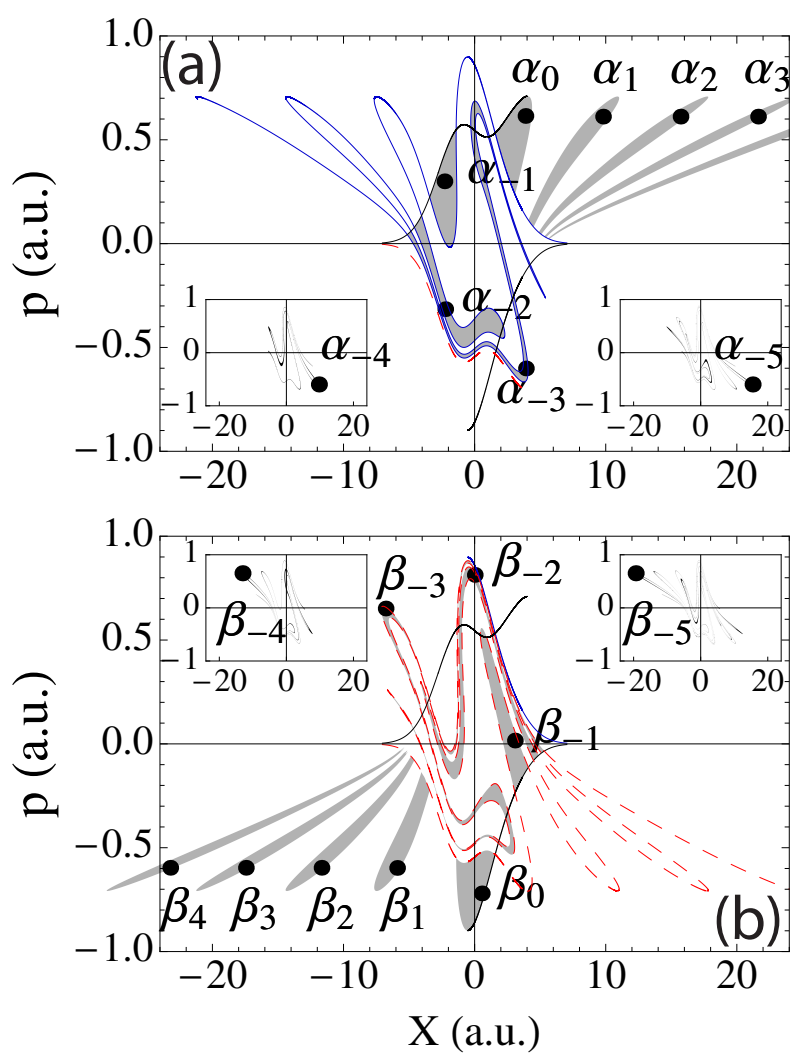

Figure A.2: (a) The points labeled $\alpha_{-5}, \ldots, \alpha_{+3}$ show the movement of a single point, $\alpha_{n}$, incident from the right for nine iterations of the PSS. Initially, the point lies in a tangle in the asymptotic region indicated by the shaded region. The point $\alpha$ takes two steps inside the fundamental area $R$ as the PSS is iterated forward in time, and eventually the entire shaded region is mapped back to the right and all points in it are reflected. (b) The points labeled $\beta_{-5}, \ldots, \beta_{+4}$ show the movement of a single point, $\beta_{n}$, incident from the left for ten iterations of the PSS. The point $\beta$ takes two steps inside the fundamental region before being reflected back to the left. 
Careful inspection of Fig. A.1.b and Figs. A.2.a and A.2.b shows that the "backward in time" filaments from the two stable manifolds become interlaced in the tendrils that extend backward in time to the upper left, and backward in time to the lower right. A particle, incident from the left, that initially lies in one of these "backward in time" tendrils can either be transmitted through the scattering region toward $x=+\infty$ or it can be reflected from the scattering region toward $x=-\infty$, depending whether it initially lies in a filament of the type shown in Fig. A.2.a or that shown in Fig. A.2.b.

In Fig. A.3.a, we show the evolution in time of a line of points initially lying between $x=-10.5$ and $x=-10.1$ for a particle incident from the left. The line of initial conditions, in the distant past, was chosen to lie across a tendril far out in the asymptotic region to the left. Fig. A.3.a shows the line of initial conditions as it is evolved forward in time, for four iterations of the PSS, and approaches the fundamental region $R$. In the next iteration the line of points will step into the fundamental region. In Fig. A.3.b, we show the time it takes various points in this line of initial conditions to leave the fundamental region (the delay time) regardless of whether the points are transmitted or reflected. The delay time forms a fractal set characteristic of this scattering process. In Fig. A.3.c, we show a plot of the number of steps taken inside $R$, by the points initially lying between $x=-10.5$ and $x=-10.1$ in Fig. A.3.a. We only show results for points that take fewer than twenty steps inside $R$. The step numbers for all initial points take values ranging from zero to infinity and form a fractal which is an image of the fractal structure of 

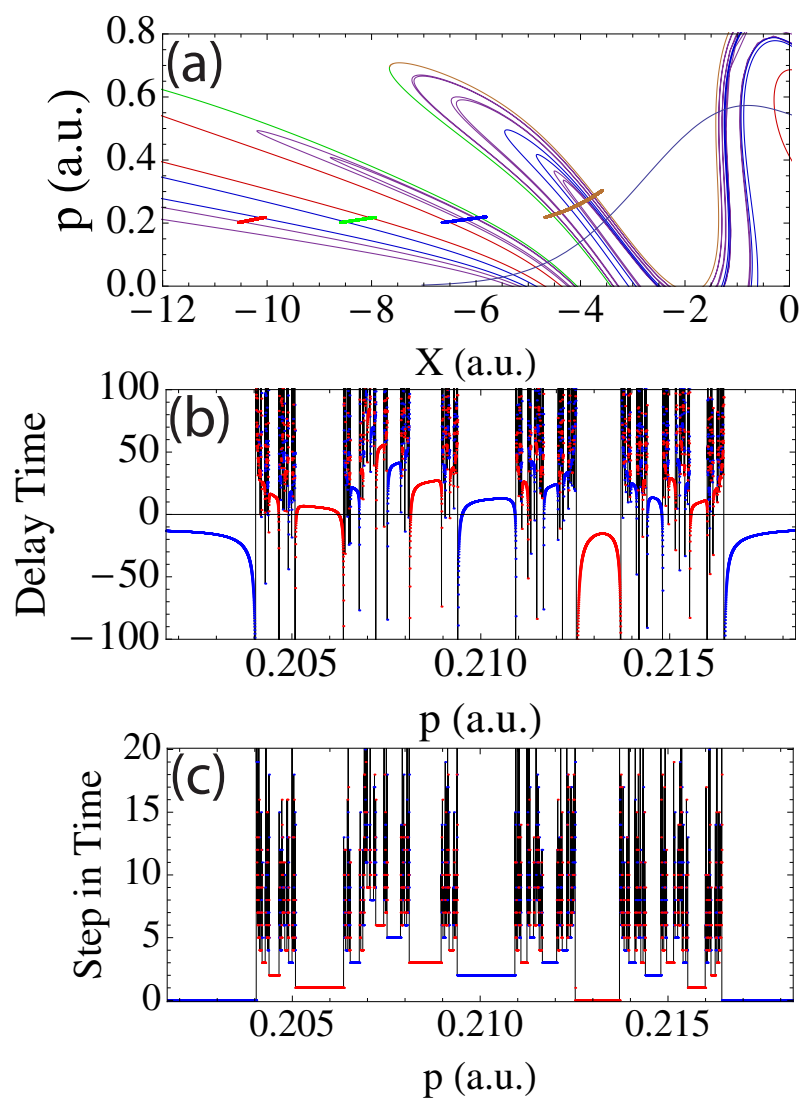

Figure A.3: (a) The evolution in time of a line of initial conditions for a particle incident from the left. The plot shows four iterations of the PSS. One more iteration would show the line of points stepping inside the fundamental region $R$. (b) The delay time (the time each point is delayed before being transmitted (red) or reflected (blue)) for the line of initial points shown in (a). (c) A plot of number of steps taken inside the fundamental region before being transmitted (red) or reflected (blue), for those trajectories that take fewer than twenty steps inside $R$. A complete plot would show step numbers ranging from one to infinity and a fractal mixed structure of transmitted and reflected points. 
the scattering region.

\section{A.4 Symbolic Dynamics}

A symbolic dynamics for this scattering process can be constructed by examining the structure of the gaps that are cut into the unstable manifolds, $\mathrm{n}-\mathrm{q}$ and $\mathrm{p}-\mathrm{m}$ by the tendrils formed by the stable manifolds $\mathrm{r}-\mathrm{p}$ and $\mathrm{s}-\mathrm{n}$ as they are iterated backward in time. In Fig. A.4.a, we show the first iteration of the stable manifolds backward in time. The shaded regions show basins of the asymptotic regions that cut through the fundamental region $R$ on this first iteration of the PSS. The shaded regions cut two segments out of the unstable manifold of $F_{L}$ and cut one segment out of the unstable manifold of $F_{R}$. We label the three remaining pieces of the unstable manifold of $F_{L}$ as "A". "B", and "C" (we follow the notation in [11]). We label the two remaining pieces of the unstable manifold of $F_{R}$ as "+" and "C". In Fig. A.4.b, we show the result of two iterations of the PSS. In this second iteration, we need to introduce two additional symbols to fully determine the symbolic dynamics. The left-most symbols of the unstable manifold of $F_{L}$ are again "A", "B", and "C" because these segments are formed in exactly that same way as in Fig. A.4.a. The segment "B-C" in Fig. A.4.a, is now cut into four segments which we label "B", "“", "+", and "C". The symbols "+" and "C" are formed in a manner similar to their formation in Fig. A.4.a. The two segments of the unstable manifold of $F_{R}$ are now cut into four segments that are labeled "A", " $\bar{A} "$, "+", and "C". The symbols "-" and "+" both branch into the symbols 

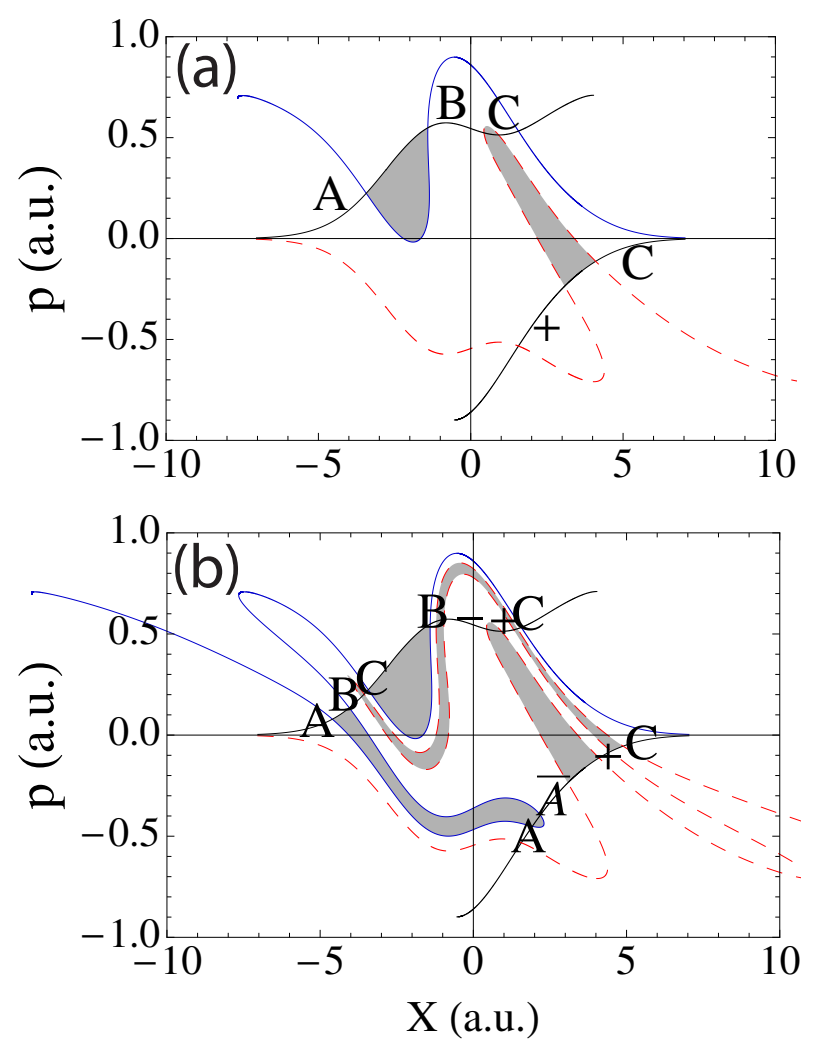

Figure A.4: (a) The first iteration of the stable manifolds backward in time. The shaded regions are basins of the asymptotic regions that cut through the fundamental region $R$ on the first iteration of the PSS. The shaded regions cut two segments out of the unstable manifold of $F_{L}$ and cut one segment out of the unstable manifold of $F_{R}$. The three remaining pieces of the unstable manifold of $F_{L}$ are labeled "A", "B", and "C". The two remaining pieces of the unstable manifold of $F_{R}$ are labeled "+" and "C". (b) The second iteration of the stable manifolds backward in time. The left-most symbols of the unstable manifold of $F_{L}$ are "A", "B", and "C" . The remaining segments are labeled "B", ".", "+", and "C". The two segments of the unstable manifold of $F_{L}$ are now cut into four segments that are labeled "A", "A", "+", and "C". 
$A$ and $\bar{A}$. When following the phase space flow under iterations of the PSS, it proved useful to distinguish "-" and "+" because they give mirror image results. However, when counting the number of symbols $A$ and $\bar{A}$ that emerge from each branch, the distinction between "-" and "+" is not important.

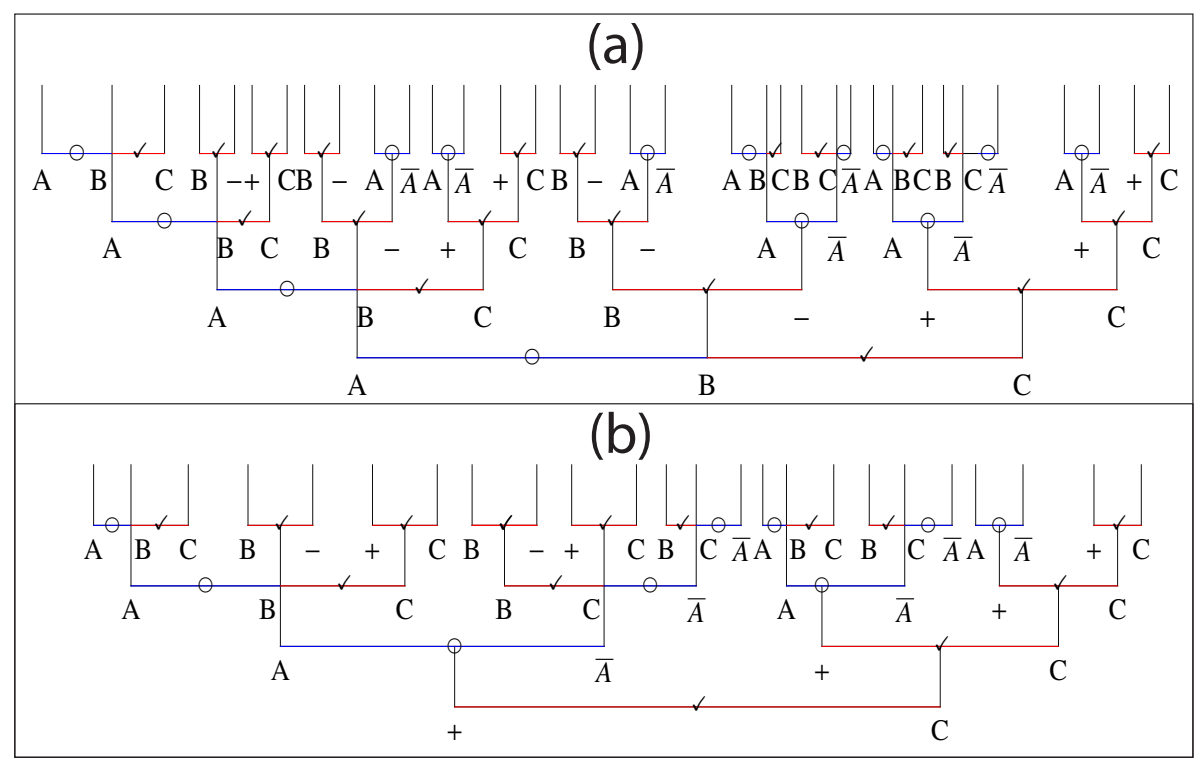

Figure A.5: (a) The branching tree for symbolic dynamics associated with scattering from the left. (b) The branching tree for scattering from the right.

Given these symbols, we can form a branching tree of symbols that show the fractal structure that emerges with successive iterations of the PSS. The branching tree for particles incident from the left is shown in Fig. 5.a for four iterations of the PSS. In Fig. A.5.a, we retain both symbols "-" and "+". Lines with symbol $\sqrt{ }$ (red online) indicate that the particle is scattered toward $F_{L}$ (is of $S_{L}$ type) while lines with symbol $\circ$ (blue online) indicates that it is scattered toward $F_{R}$ (is of $S_{R}$ type). The branching tree for particles 
incident from the right is shown in Fig. A.5.b. We can now represent the branching trees in terms of a transfer matrix $\mathbf{T}_{6}$ that acts on a column matrix $\mathbf{s}_{6}=(A, B, C,+,-, \bar{A})^{T}$ (T denotes transpose $)$ formed with the six symbols that comprise the symbolic dynamics for this system. The transfer matrix $\mathbf{T}_{6}$ is given in Eq. (3),

$$
\mathbf{T}_{6}=\left(\begin{array}{cccccc}
1 & 0 & 0 & 1 & 1 & 0 \\
1 & 1 & 0 & 0 & 0 & 1 \\
1 & 0 & 1 & 0 & 0 & 1 \\
0 & 0 & 1 & 0 & 0 & 0 \\
0 & 1 & 0 & 0 & 0 & 0 \\
0 & 0 & 0 & 1 & 1 & 1
\end{array}\right), \quad \mathbf{T}_{5}=\left(\begin{array}{lllll}
1 & 0 & 0 & 1 & 0 \\
1 & 1 & 0 & 0 & 1 \\
1 & 0 & 1 & 0 & 1 \\
0 & 1 & 1 & 0 & 0 \\
0 & 0 & 0 & 1 & 1
\end{array}\right)
$$

If we do not distinguish the symbols "-" and "+" and everywhere replace symbol "-" with "+", then the transfer matrix becomes a $5 \times 5$ matrix $\mathbf{T}_{5}$ which is also shown in Eq. (3). The transfer matrix $\mathbf{T}_{5}$ acts on a column matrix $\mathbf{s}_{5}=(A, B, C,+, \bar{A})^{T}$

The transfer matrices $\mathbf{T}_{\alpha}(\alpha=5,6)$ are not self adjoint. Therefore, $\mathbf{T}_{\alpha}$ will have $\alpha$ left eigenvectors $\boldsymbol{\psi}_{j}^{(\alpha)}, \alpha$ right eigenvectors $\boldsymbol{\phi}_{j}^{(\alpha)}$, and $\alpha$ eigenvalues $\lambda_{j}^{(\alpha)}$, where $j=1, \ldots, \alpha$. The eigenvectors satisfy orthonormality conditions $\boldsymbol{\psi}_{j}^{(\alpha)} \cdot \boldsymbol{\phi}_{j^{\prime}}^{(\alpha)}=\delta_{j, j^{\prime}}$. In terms of these eigenvalues and left and right eigenvectors, the transfer matrix can be written $\mathbf{T}_{\alpha}=\sum_{j=1}^{\alpha} \lambda_{j}^{(\alpha)} \boldsymbol{\phi}_{j}^{(\alpha)} \cdot \boldsymbol{\psi}_{j}^{(\alpha)}$. For both $\alpha=5$ and $\alpha=6$, there is one eigenvalue with value $\lambda_{1}^{(\alpha)}=2.3146$, while for all the other eigenvalues $\operatorname{Re}\left[\lambda_{j}^{(\alpha)}\right] \leq 1, j=2, \ldots, \alpha$. Therefore, when the transfer matrix acts $n$ times, we obtain $\mathbf{T}_{\alpha}^{n} \rightarrow\left(\lambda_{1}^{(\alpha)}\right)^{n} \boldsymbol{\phi}_{1}^{(\alpha)} \cdot \boldsymbol{\psi}_{1}^{(\alpha)}$ as $n \rightarrow \infty$. The left and 
right eigenvectors of $\mathbf{T}_{6}$ and $\mathbf{T}_{5}$ are

$$
\begin{gathered}
\boldsymbol{\psi}_{1}^{(6)}=(0.441,0.290,0.290,0.381,0.381,0.441) \\
\boldsymbol{\phi}_{1}^{(6)}=(0.441,0.671,0.671,0.290,0.290,0.441)^{T}
\end{gathered}
$$

and

$$
\begin{gathered}
\boldsymbol{\psi}_{1}^{(5)}=(0.441,0.290,0.290,0.763,0.441) \\
\phi_{1}^{(5)}=(0.441,0.671,0.671,0.580,0.441)^{T}
\end{gathered}
$$

respectively. Note that, since the transfer matrices are known exactly, the accuracy of these numbers is only limited by the accuracy of the matrix solver.

We can now reproduce the number of each of the symbols "A", "B", "C", "+", " -", " $\bar{A}$ " at level $n$ in the branching tree for scattering from the left by allowing the transfer matrix to act $n-1$ times on the initial partition $\mathbf{s}_{1}^{L}=$ $(1,1,1,0,0,0)^{T}$ of the unstable manifold of $F_{L}$ (see Fig. A.4.a). We obtain $\mathbf{T}_{6}^{n-1} \cdot \mathbf{s}_{1}^{L}=1.021(2.315)^{n-1} \boldsymbol{\phi}_{1}^{(6)}$. Similarly, if we allow the transfer matrix to act $n-1$ times on the initial partition $\mathbf{s}_{1}^{R}=(0,0,1,1,0,0)^{T}$ of the unstable manifold of $F_{R}$ (see Fig. 4.a) for scattering from the right, we obtain $\mathbf{T}_{6}^{n-1} \cdot \mathbf{s}_{1}^{R}=$ $0.671(2.315)^{n-1} \boldsymbol{\phi}_{1}^{(6)}$. (A similar analysis can be performed on $\mathbf{T}_{5}$.)

It only takes a few steps up the branching tree for scattering from the left, or from the right, for the fractional distribution of symbols "A", "B", "C", "+", " -", " $\bar{A} "$ to be determined by the fractional distribution of symbols in the right eigenvector $\phi_{1}$. Thus, for both incident directions, the branching trees, as regards the fraction of each type of symbol present, become the same. 
The fractions are $f_{A}=0.157, f_{B}=0.239, f_{C}=0.239, f_{+}=0.103, f_{-}=0.103$, and $f_{\bar{A}}=0.157$.

We now can use this information, and the information in Fig. A.5.b, to obtain a rough estimate of the likelihood that an incident particle, that gets caught up in the fractal structure, gets transmitted or reflected. First remember that $\sqrt{ }(\mathrm{o})$ indicates a gap containing trajectories that ultimately travel to $-\infty$ and are of $S_{L}$ type (travel to $+\infty$ and are of $S_{R}$ type). From Fig. A.5.b, we see that intervals of type "A" and " $\bar{A}$ " in an unstable manifold, in the next iteration of the map, will contain one $S_{L}$ gap and one $S_{R}$ gap. Intervals of type "B" and "C", in the next iteration of the map, will contain two $S_{L}$ gaps. Intervals of type "+" and "-_, in the next iteration of the map, will contain two $S_{R}$ gaps. The fraction of $S_{L}$ gaps, in the next iteration of the map, is 0.636 and the fraction of $S_{R}$ gaps is 0.364 . Thus, for particles incident from the left, $36.4 \%$ of the gaps get transmitted and $63.6 \%$ of the gaps get reflected. For particles incident from the right, $63.6 \%$ of the gaps get transmitted and $36.4 \%$ get reflected.

For a given line of initial points that cross a tendril in the asymptotic region, the percentage of gaps transmitted or reflected gives an indication of the asymmetry of the scattering process but it does not give the actual value for the percentage of initial points transmitted or reflected. Lines of initial points can cross tendrils in the asymptotic region in different places, at different angles, etc. The stepping time plots, such as that shown in Fig. A.3.c, do give that information because they distinguish the initial points being 
scattered to the right ( $S_{R^{-}}$type and colored blue online) from the initial points being scattered to the left ( $S_{L}$-type and colored red online). By adding up the length of the $S_{R}$ lines and the $S_{L}$ lines in Fig. A.3.b, we find that, for the line of initial conditions shown in Fig. A.3, of those crossing the tendril, 44.24\% are transmitted and $55.76 \%$ are reflected.

\section{A.5 Conclusions}

The PSS that we use (plot $p$ and $x$ each time $\phi=\pi / 2+n 2 \pi$, where integer $n=0,1,2, .$.$) to analyze the scattering dynamics corresponds to a par-$ ticular phase of the radiation field at time $t=0$ when we begin to follow our trajectories. If the phase of the field is different at time $t=0$ then the asymmetric scattering properties will differ in detail but will still be asymmetric. The procedure described here can be applied to any choice of initial phase of the radiation field. The symbolic dynamics of the scattering process, and the resulting transfer matrix, can provide an important tool for disentangling reflection from transmission in such scattering processes. 


\section{Appendix B}

\section{Fractal Dimension of 2D and 3D $\mathrm{HOCl}$}

In Fig. B.1.a, we again plot $p_{R}$ versus $\chi$, as in Fig. 3.4.b, but now with 5000 data points for $\chi$, so that more of the fractal structure emerges. In Figs. B.1.b and B.1.c, we enlarge the fractal regions on the left and right of Fig. B.1.a. In Fig. B.2 we show plots of $p_{R}$ versus $\chi$ for the $2 \mathrm{D}$ model of $\mathrm{HOCl}$ with the $\mathrm{HO}$ bond held fixed at its equilibrium displacement. Comparing Figs. B.1.a and B.2.a, we see that the scattering dynamics of the two cases is very similar, although there are also small differences. In both Fig. B.1.b and Fig. B.2.b there are "mirror" points in the discontinuous regions, located directly under the letters B, A, and C. The structures of local regions on either side of these mirror points are approximate mirror images of each other, although the right-hand side is compressed relative to the left-hand side. The large scale similarities between the plots in Figs. B.1 and B.2 indicate that the 2D model of $\mathrm{HOCl}$ is fairly good at reproducing the regions of continuity and discontinuity in the $3 \mathrm{D}$ dynamics, as long as the $\mathrm{HO}$ vibration is close to its minimum value initially.

Although the plots in Figs. B.1 and B.2 are very similar, it is clear that small parts of the intervals of continuity in the $2 \mathrm{D}$ case contain singular 

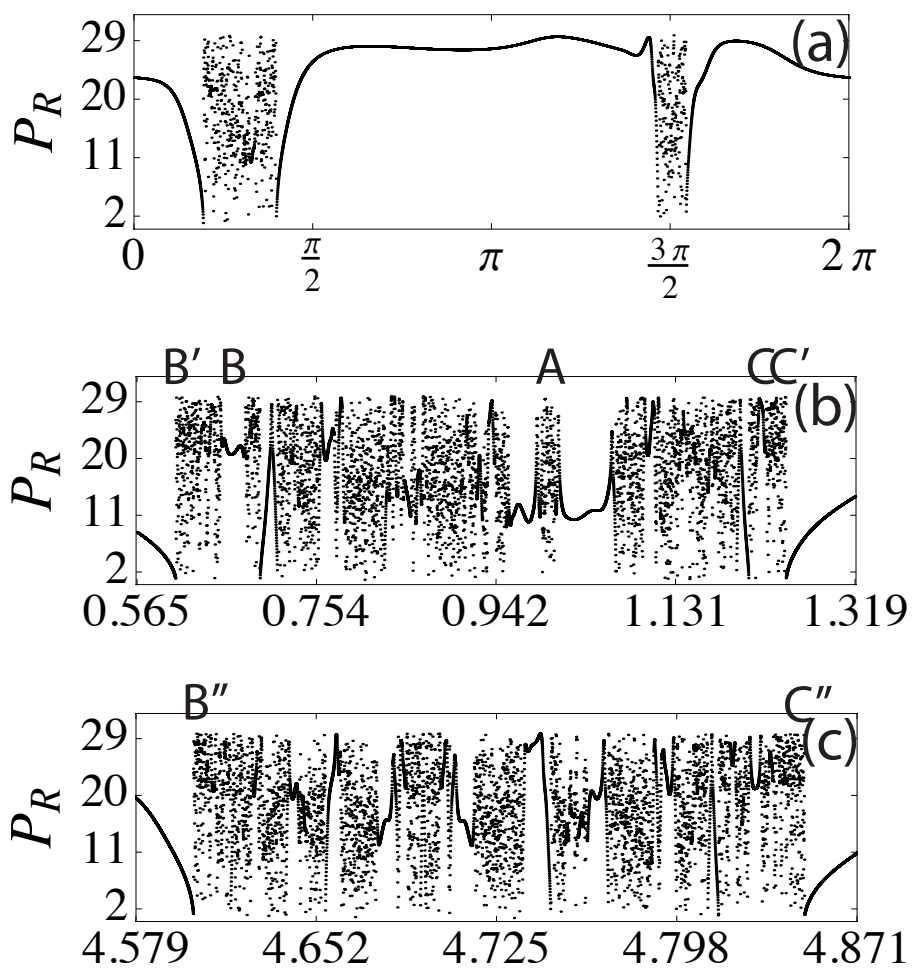

Figure B.1: Scattering dynamics of the 3D Cl-HO system. (a) Magnification of Fig. 3.4.b (5000 data points for $\chi$ ). (b) Magnification of the left discontinuous region of Fig. B.1.a. (c) Magnification of the right discontinuous region of Fig. B.1.a. $\left(p_{R}\right.$ in dimensionless units and $\chi$ in radians.) 
points and become discontinuous in the 3D case. This is seen most clearly when comparing the mirror points $\mathrm{B}$ and $\mathrm{A}$ in Figs. B.1.b and B.2.b, respectively. These mirror-like structures repeat and can be found embedded locally in these plots as one goes to ever finer scales in the phase space, so there appears to be a fractal structure embedded in the phase space. Using the "box-counting" technique, we have computed the fractal dimension of the intervals of discontinuity that appear to the left and right of Figs. B.1.a and B.2.a. For the interval of discontinuity on the left (enlarged in Figs. B.1.b and B.2.b), we find that the intervals of discontinuity for $3 \mathrm{D}$ have a fractal dimension of 0.88 , while the intervals of discontinuity for $2 \mathrm{D}$ have a fractal dimension of 0.80 . For the interval of discontinuity on the right (enlarged in Figs. B.1.c and B.2.c), we find that the intervals of discontinuity for 3D have fractal dimension of 0.91 , while the intervals of discontinuity for $2 \mathrm{D}$ have a fractal dimension of 0.86 .

In Fig. B.3, we show the time it takes for $\mathrm{Cl}$ to leave the asymptotic region at $R=12$ d.u., interact with $\mathrm{HO}$, and finally return to the asymptotic region at $R=12 \mathrm{~d}$.u. for a range of values of initial phase $\chi$ in the neighborhood of the mirror point at "B". Note that Fig. B.3 shows the running times $\tau$ for $\mathrm{Cl}$ (time for $\mathrm{Cl}$ to "run" from its initial position at $R=12$ d.u., interact with HO (delay time), and then "run" back to its initial position). The "running time" for a free particle to travel a distance $2 R$ is approximately 3,000 d.u. Since the initial momentum of $\mathrm{Cl}$ is the same for all initial values of $\chi$, the times shown in Fig. B.3 differ from the delay time (the interaction time) 

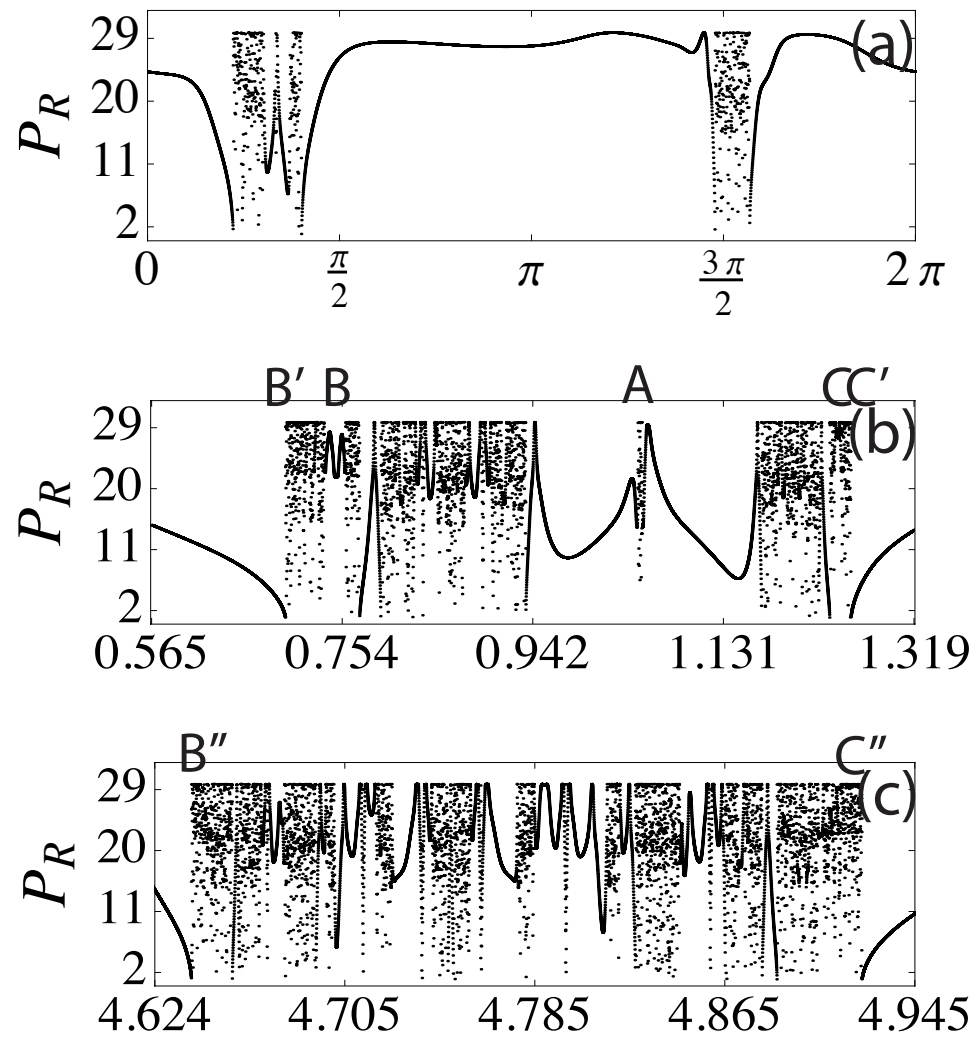

Figure B.2: Scattering dynamics of the 2D Cl-HO system. (a) A plot of $p_{R}$ versus $\chi$ for the case is which the bond length of the $\mathrm{HO}$ dimer is held fixed at the equilibrium value $r_{e q}=1.8389$ d.u., $p_{\theta}=5.92$ d.u., and the total energy is fixed at $E=1.231$ d.u. $=25,000 \mathrm{~cm}^{-1}$ (5000 data points for $\left.\chi\right)$. (b) Magnification of left discontinuous region in Fig. 4.a. (c) Magnification of the right discontinuous region in Fig. 4.a. $\left(p_{R}\right.$ in dimensionless units and $\chi$ in radians.) 
only by a constant factor. The running time for the 3D case for $\psi=\pi$ and 0.584 d.u. $\leq \chi \leq 0.712$ d.u. is shown in Fig. B.3.a. In Fig. B.3.b we show the running time for the $2 \mathrm{D}$ case for the interval 0.679 d.u. $\leq \chi \leq 0.792$ d.u.. These plots are again very similar, and they show the same fractal structure as the corresponding plots for $p_{R}$ versus $\chi$. However, the running time plots do have some differences which show that the $\mathrm{HO}$ vibration can become involved in the scattering process, even if it has very little energy initially.
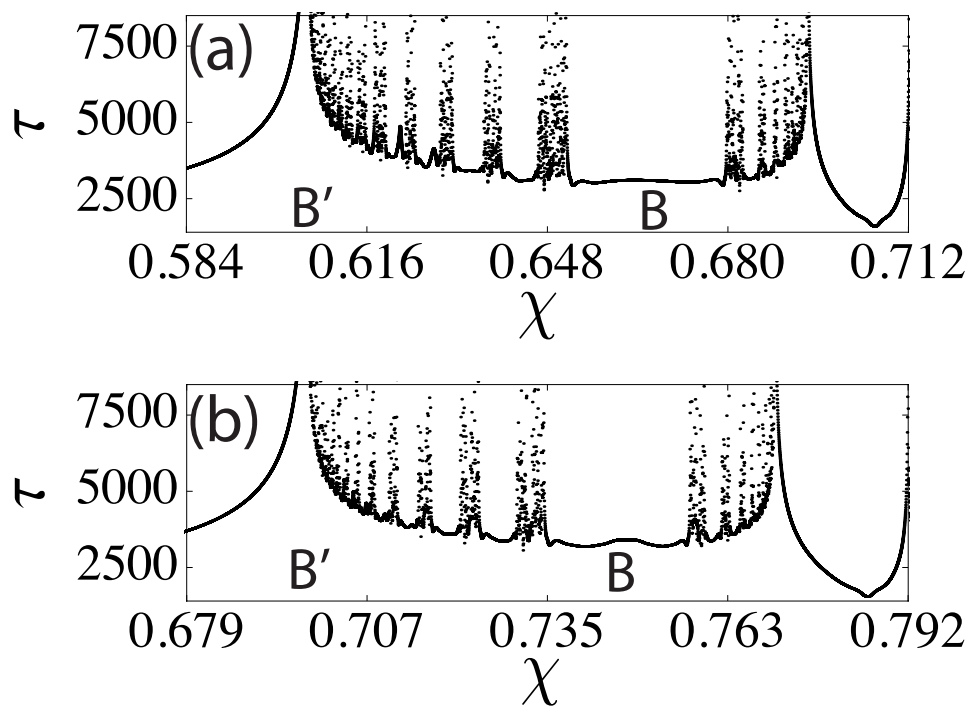

Figure B.3: Running time plots for values of $\chi$ in the neighborhood of the mirror point B. Note that these plots contain the time for $\mathrm{Cl}$ to travel from $R=12 \mathrm{~d} . \mathrm{u}$. to the reaction region, then interact with $\mathrm{HO}$ and return to $R=12$ d.u.. (a) 3D molecule. (b) 2D molecule. ( $\tau$ in dimensionless units and $\chi$ in radians.) 


\section{Bibliography}

[1] Stavros C Farantos, Reinhard Schinke, Hua Guo, and Marc Joyeux. Energy localization in molecules, bifurcation phenomena, and their spectroscopic signatures: The global view. Chemical reviews, 109(9):4248-4271, 2009.

[2] R Schinke, J Suarez, and SC Farantos. Communication: Photodissociation of n2o-frustrated nn bond breaking causes diffuse vibrational structures. Journal of Chemical Physics, 133(9):091103, 2010.

[3] Frederic Mauguiere, Stavros C Farantos, Jaime Suarez, and Reinhard Schinke. Non-linear dynamics of the photodissociation of nitrous oxide: Equilibrium points, periodic orbits, and transition states. The Journal of chemical physics, 134(24):244302, 2011.

[4] R Schinke. Photodissociation of n2o: Potential energy surfaces and absorption spectrum. The Journal of chemical physics, 134(6):064313, 2011.

[5] Alex M Barr, Kyungsun Na, and LE Reichl. Quasibound states in a chaotic molecular system. Physical Review A, 83(6):062510, 2011.

[6] Alex M Barr and LE Reichl. Wigner-smith delay times and the nonhermitian hamiltonian for the hocl molecule. Fortschritte der Physik, 61(2-3):59-65, 2013. 
[7] Linda Reichl. The transition to chaos: conservative classical systems and quantum manifestations. Springer Science \& Business Media, 2004.

[8] Stephen Wiggins. Normally hyperbolic invariant manifolds in dynamical systems. Number 105. Springer Science \& Business Media, 1994.

[9] C Jung and HJ Scholz. Cantor set structures in the singularities of classical potential scattering. Journal of Physics A: Mathematical and General, 20(12):3607, 1987.

[10] B Ruckerl and C Jung. Scaling properties of a scattering system with an incomplete horseshoe. Journal of Physics A: Mathematical and General, $27(1): 55,1994$.

[11] Agapi Emmanouilidou, C Jung, and LE Reichl. Classical scattering for a driven inverted gaussian potential in terms of the chaotic invariant set. Physical Review E, 68(4):046207, 2003.

[12] C Jung and A Emmanouilidou. Construction of a natural partition of incomplete horseshoes. Chaos: An Interdisciplinary Journal of Nonlinear Science, 15(2):023101, 2005.

[13] A Emmanouilidou and C Jung. Partitioning the phase space in a natural way for scattering systems. Physical Review E, 73(1):016219, 2006.

[14] Alex M Barr, Kyungsun Na, LE Reichl, and Christof Jung. Chaotic scattering in a molecular system. Physical Review E, 79(2):026215, 2009. 
[15] Yi-Der Lin, Alex Barr, Kyungsun Na, and Linda E Reichl. Fractal scattering in a radiation field. Physical Review E, 83(5):056217, 2011.

[16] J Weiss, J Hauschildt, S Yu Grebenshchikov, R Düren, R Schinke, J Koput, S Stamatiadis, and SC Farantos. Saddle-node bifurcations in the spectrum of hocl. The Journal of Chemical Physics, 112(1):77-93, 2000.

[17] C Jung, O Merlo, TH Seligman, and WPK Zapfe. The chaotic set and the cross section for chaotic scattering in three degrees of freedom. New Journal of Physics, 12(10):103021, 2010.

[18] Yi-Der Lin, Alex M Barr, LE Reichl, and Christof Jung. Fractal scattering dynamics of the three-dimensional hocl molecule. Physical Review E, 87(1):012917, 2013.

[19] Yi-Der Lin, LE Reichl, and Christof Jung. The vibrational dynamics of 3d hocl above dissociation. The Journal of Chemical Physics, 142:124304, 2015.

[20] Marc Joyeux, Dominique Sugny, Maurice Lombardi, Remy Jost, Reinhard Schinke, Sergei Skokov, and Joël Bowman. Vibrational dynamics up to the dissociation threshold: A case study of two-dimensional hocl. The Journal of Chemical Physics, 113(21):9610-9621, 2000.

[21] J Hauschildt, J Weiß, C Beck, S Yu Grebenshchikov, R Düren, Reinhard Schinke, and J Koput. Unimolecular dissociation of hocl: unex- 
pectedly broad distribution of rate constants. Chemical physics letters, 300(5):569-576, 1999.

[22] Sergei Skokov and Joel M Bowman. Complex 12 calculation of the variation of resonance widths of hocl with total angular momentum. The Journal of chemical physics, 111(11):4933-4941, 1999.

[23] Hamse Y Mussa and Jonathan Tennyson. Calculating quasi-bound rotationvibrational states of hocl using massively parallel computers. Chemical physics letters, 366(5):449-457, 2002.

[24] Max Born. The mechanics of the atom. 1960.

[25] C Jung, C Mejia-Monasterio, O Merlo, and TH Seligman. Self-pulsing effect in chaotic scattering. New Journal of Physics, 6(1):48, 2004.

[26] Christian Dembowski, B Dietz, H-D Gräf, A Heine, T Papenbrock, A Richter, and C Richter. Experimental test of a trace formula for a chaotic threedimensional microwave cavity. Physical review letters, 89(6):064101, 2002.

[27] Hoshik Lee and LE Reichl. Dicke effect in a multiripple electron waveguide. Physical Review B, 77(20):205318, 2008.

[28] Christian Dembowski, B Dietz, T Friedrich, H-D Gräf, A Heine, C MejíaMonasterio, M Miski-Oglu, A Richter, and TH Seligman. First experimental evidence for quantum echoes in scattering systems. Physical review letters, 93(13):134102, 2004. 
[29] Holger Waalkens, Roman Schubert, and Stephen Wiggins. Wigner's dynamical transition state theory in phase space: classical and quantum. Nonlinearity, 21(1):R1, 2008.

[30] Holger Waalkens and Stephen Wiggins. Geometrical models of the phase space structures governing reaction dynamics. Regular and Chaotic Dynamics, 15(1):1-39, 2010.

[31] R Schubert, H Waalkens, and S Wiggins. A quantum version of wigners transition state theory. Few-Body Systems, 45(2-4):203-206, 2009.

[32] G Barton. Quantum mechanics of the inverted oscillator potential. Annals of Physics, 166(2):322-363, 1986.

[33] Christof Jung and H-J Scholz. Chaotic scattering off the magnetic dipole. Journal of Physics A: Mathematical and General, 21(10):2301, 1988.

[34] Bruno Eckhardt. Irregular scattering. Physica D: Nonlinear Phenomena, 33(1):89-98, 1988.

[35] Christopher Jung and Thomas H Seligman. Integrability of the s-matrix versus integrability of the hamiltonian. Physics reports, 285(3):77-141, 1997.

[36] Agapi Emmanouilidou and LE Reichl. Floquet scattering and classicalquantum correspondence in strong time-periodic fields. Physical Review A, 65(3):033405, 2002. 
[37] Kyungsun Na, Daungruthai Jarukanont, and LE Reichl. Dynamics of quasibound state formation in the driven gaussian potential. Physical Review E, 77(4):046208, 2008.

[38] Hendrik Anthony Kramers. Collected scientific papers. North-Holland Publishing Company, 1956.

[39] Walter C Henneberger. Perturbation method for atoms in intense light beams. Physical Review Letters, 21(12):838, 1968.

[40] Herbert Goldstein. Classical mechanics. Pearson Education India, 1957.

[41] Guanhua Yao and Shih-I Chu. Complex-scaling fourier-grid hamiltonian method. iii. oscillatory behavior of complex quasienergies and the stability of negative ions in very intense laser fields. Physical Review A, 45(9):6735, 1992.

[42] M Marinescu and M Gavrila. First iteration within the high-frequency floquet theory of laser-atom interactions. Physical Review A, 53(4):2513, 1996.

[43] AS Fearnside, RM Potvliege, and Robin Shakeshaft. Light-induced states of $\mathrm{h}$ and h-, shadow states, and the dressed potential. Physical Review A, 51(2):1471, 1995.

[44] Z Kovács and L Wiesenfeld. Topological aspects of chaotic scattering in higher dimensions. Physical Review E, 63(5):056207, 2001. 